$$
\text { COO-2456-46 }
$$

Report No. 71

\title{
Columbia alnibersity
} in the city of 3ew Dork

\author{
Hager. \\ SURVEY OF ITNFAR MHD STABILITY \\ IN TOKAMAK CONFIGURATIONS
}

by

Masahiro Wakatani

1977

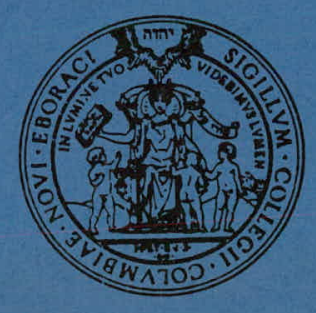

Plasma Laboratory

School of Engineering and Applied Science Columbia University

New York, N. Y. 10027 


\section{DISCLAIMER}

This report was prepared as an account of work sponsored by an agency of the United States Government. Neither the United States Government nor any agency Thereof, nor any of their employees, makes any warranty, express or implied, or assumes any legal liability or responsibility for the accuracy, completeness, or usefulness of any information, apparatus, product, or process disclosed, or represents that its use would not infringe privately owned rights. Reference herein to any specific commercial product, process, or service by trade name, trademark, manufacturer, or otherwise does not necessarily constitute or imply its endorsement, recommendation, or favoring by the United States Government or any agency thereof. The views and opinions of authors expressed herein do not necessarily state or reflect those of the United States Government or any agency thereof. 


\section{DISCLAIMER}

Portions of this document may be illegible in electronic image products. Images are produced from the best available original document. 
SURVEY OF LINEAR MHD STABILITY

IN

TOKAMAK CONFIGURATIONS

Thi NOTICE

This report was prepared as an account of work the United States ned States Government. Neither Rescarch and Development Administontion Eores Energy their employees, nor any of theis tubcontractors, or their employees, makes any warranty, express or implied, or assumes any legal berify or responsibily for the accuracy, completenes

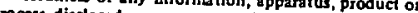
infringe privately owned rights.

Masahiro Wakatani*

(Columbia University, visiting staff)

*Institute of Plasma Physics

Nagoya University, Nagoya

Japan

Work Supported by ERDA Contract EY-76-S-02-2456 
TABLE OF CONTENTS

1. Introduction

References for Section 1

2. MHD Stability of External Kink Modes 5 Table I: Classifications : 6

2-1. Low Beta Tokamaks 7

2-1-1. Circular Cross-Section Tokamak 8

2-1-2. Non-Circular Cross-section Tokamak

2-2. High Beta Tokamaks

2-2-1. High Beta Tokamaks and Skin Current

2-2-2. High Beta Tokamak with Diffuse Current Profile

References for section 2

Figures for section 2

3. MHD Stability of Internal Kink Modes

References for section 3

Figures for section 3 


\section{Introduction}

Recently, low $\beta$ tokamaks, which are axisymmetrịc toroidal plasmas, have succeeded in obtaining energy confinement time $\tau_{E} \sim 40 \mathrm{msec}$, electron density $\mathrm{n} \mathrm{e}^{10^{14}} \mathrm{~cm}^{-3}$, ion temperature $T_{i} \sim_{1} \sim 2 \mathrm{kev}$ and electron temperature $\mathrm{T}_{\mathrm{e}} \sim^{2} \sim_{3} \mathrm{kev}$, for a safety factor $\left.q(a) \sim 4 \sim_{6},{ }^{j}, 2^{2}\right)$ These data are significant milestones for controlled thermonuclear reactor research; however, the ratio of plasma pressure to magnetic pressure, $\beta$, is less than $1 \%$. From the point of view of fusion reactor economy, it is desirable to operate tokamak reactors at high $\beta$. The usual reason is magnetic energy utilization $P_{T} \alpha^{\alpha} B^{2}$, where $P_{T}$ is the thermonuclear power and $B$ is the magnetic field. ${ }^{3)}$ From a plasma transport point of view, one expects operation at the highest possible density or at critical $\beta$ to be favorable for suppression of trapped particle mode transport. ${ }^{4}$ )

Several methods are considered to raise $\beta$ values in tokamak plasmas. The relation between total beta $\beta$ and poloidal beta $\beta_{P}$ is

$$
\beta=\beta_{p} \frac{B_{p}^{2}}{B_{p}^{2}+B_{T}^{2}}
$$

where ${ }_{P}$ and $B_{T}$ are the poloidal and toroidal magnetic fields respectively. In a tokamak of large aspect ratio, $a / R \ll 1$, $B_{p}$ must be smaller than $B_{T}$ to satisfy the Kruskal-Shafranov limit, $q=a / R{ }_{T} /_{B_{p}}>1$ and the relation (1) reduces to 


$$
\beta=\beta_{p}\left(\frac{a}{R}\right)^{2} \frac{1}{q}^{2}
$$

From (2), to obtain a higher $\beta$, it is desirable to raise $\beta_{p}$ and lower $R / a$ and $q$. Experimentally $\beta_{p} \sim_{0.5} \sim_{1}$ is found in Ohmic-heated tokamaks and $\tau_{E}$ becomes worse for $q<3$. If $\beta_{p} \sim 1$ cannot be exceeded, higher $\beta$ can be reached by raising the poloidal magnetic field $B_{p} \sim_{B}$ from the relation (1). Writing the Kruskal-shafranov linit as

$$
q=\frac{B_{T}}{B_{p}} \frac{\ell_{p}}{\ell_{T}}>1
$$

$\left(\ell_{T}\right.$ and $\ell_{j}$ are lengths of the torroidal and poloidal circumference of the torus, respectively), we see that, for fixed value of $B_{T}$, larger values of $B_{p}$ are permitted only if the ratio $l_{\mathrm{P}} / l_{\mathrm{T}}$ is increased. This is done most effectively by elongating the cross section parailel to the major axis. The research of non-circular tokamaks is pursued to increase the $B$ of tokamak plasmas.

On the other hand, Friedberg and Haas ${ }^{5}$ ) studied the regime, $\beta n, a / R$ and $\beta_{p} \backsim R / a$, known as 'high $\beta$ ordering'. In this regime the confinement of the plasma is mainly due to the toroidal magnetic field. Critical $\beta$ determined by MHD stability is calculated by use of the high $\beta$ ordering.

Recently MHD equilibria with $\beta_{p} \geq R / a$ and no plasma current reversals are found by the concept of flux-conserving tokamak (FCT).6) Flux conservation may be possible by use of 
a rapid high-power neutral beam injection heating. As one approach to obtain a high $\beta$ tokamak, FCT is studied in ORNL. ${ }^{6}$ )

In high $\beta$ tokamaks, the most important problem may be MHD stability of external kink modes and internal kink modes, and attainable maximum $\beta$ will be determined by these instabilitico. When we study MHD stability, we separate the problem into the calculation of equilibrium configurations which represent the near steady state behavior and the investigation of small perturbations about these equilioria. These are described by the linearized MHD equations. In $\$ 2$ we survey the results found by MнD stability studies for both low $B$ tokamaks and high $B$ tokamaks. Here our concerns are of stability against kink-ballooning modes in equilibria surrounded by vacuum or a layer of force-free currents. In $\$ 3$ internal kink modes and the relation to interchange (or flute) modes, which should be considered after external kink modes are surpressed, are surveyed. The internal kink mode is important for the stability of diffuse current profile. It is also related to the important ballooning mode. 
4

References of $\$ 1$

1. D. Grove et. al., 6th IAEA Conference on Plasma Physics and Controlled Nuclear Fusion Research (Berchtesgaden). $(1976), \mathrm{CN}-35 / \mathrm{A} 2$.

2. G. Bowman et. al., in Proc. 7th European Conf. on Controlled Fusion and Plasma Physics (Lausanne, 1975), vol. IT, p. 14 .

3. F, Rice, Rev, Mod. Phys, $47 ; 7$ (1975).

4. D.J. Simar and J.P. Friedberg in Proc. 3rd Topical Conference on High $\beta$ Pulsed Plasmas (Culham) 1975.

5. J.P. Freidberg and F.A. Haas, Phys. Fluids 16.1909 (1973).

6. J.F. Clarke in Bulletin APS 20, 1BI (1975). 


\section{MHD Stability of External Kink Modes}

Here we survey MHD stability analyses of external kink instabilities (or kink-ballooning instabilities) in tokamak plasma. The kink modes have large growth rates characterized with the transit time of poloidal Alfven velority $B_{p}(a) / \sqrt{4 \pi \rho_{0}}$ and eigenfunctions (or perturbations) expended over the plasma column. The critical $\beta$, under which a plasma column is stable to external kink modes, may give stringent conditions on most experiments.

The MHD stability analyses of axisymmetric toroidal plasmas may be classified in two groups, low $\beta$ tokamaks and high $\beta$ tokamaks, according to their ordering and/or assumptions used in the analysis. Each group is divided into three types by their equilibrium configurations, circular cross-sections, elliptical cross-sections, and other cross-sections (race track, rectangular or doublet). Since stability of external kink modes with $m \geq 2$ ( $m$ is poloidal mode number) depends critically on the plasma current profile; we consider 3 cases; pure skin current, uniform current, and more general diffuse current profiles.

Properties of external kink instabilities are related to the equilibrium configurations. Complete MHD stability analyses in a real tokamak system are very complicated. In order to make analyses analytically tractable, we usually consider a simplified configuration retaining essential properties of the original one. The most important 


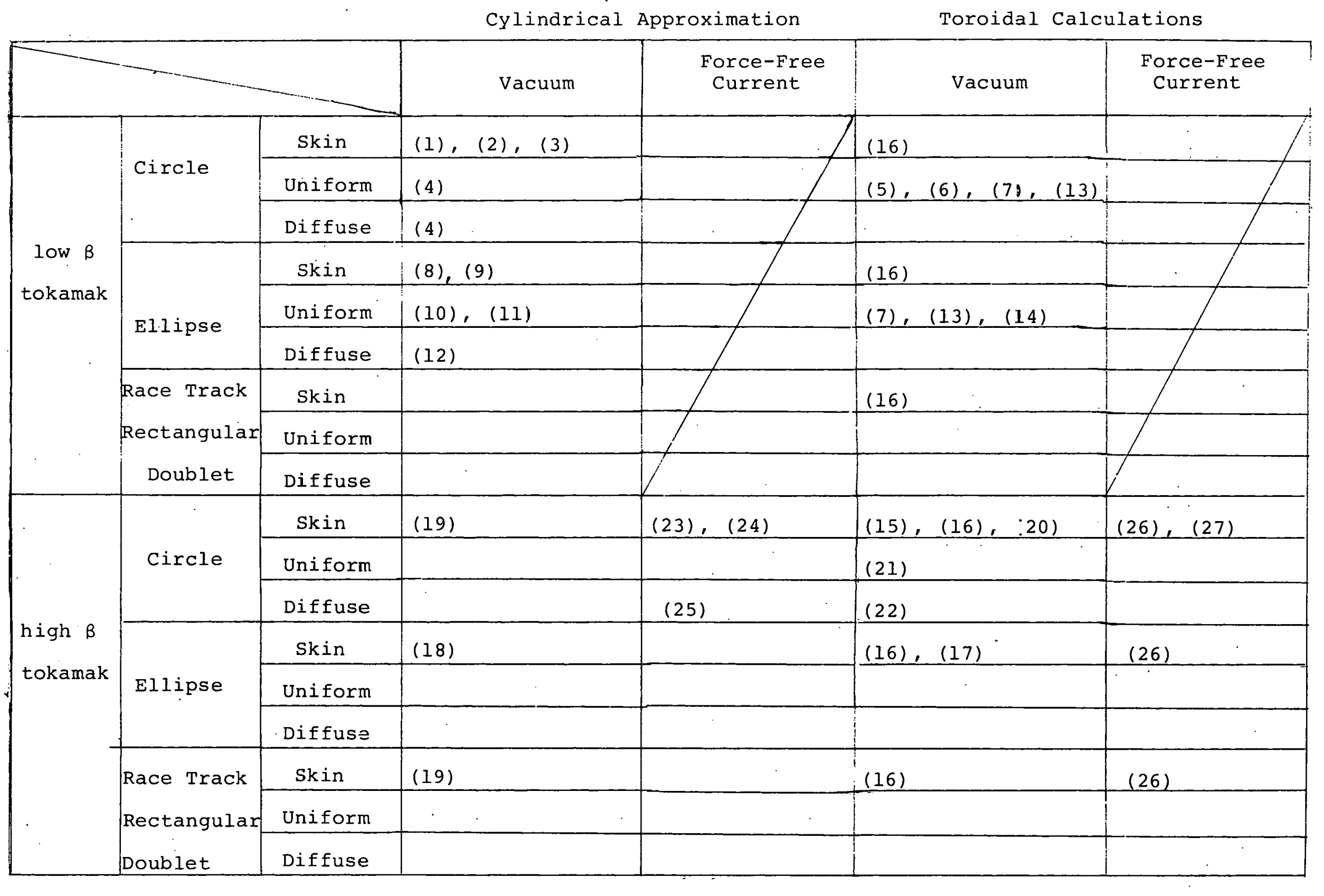


approximation to a toroidal plasma with a minor radius a and a major radius $R$ is to consider a cylindrical plasma column of radius $a$ and periodic length $L=2 \pi R$. The famous stability condition, 1.e. Kruskal'1)- Shafranov' ${ }^{2}$ limit, is obtained by cylindrical approximation, Here we divide MHD analyses of external kink modes into two groups, 'cylindrical approximation' and 'toroidal calculation.'

It was pointed out by the Jutphaas group 23 ), 24) that a force-free current layer on the outside of a plasma column has stabilizing effects on externai kink instabilities. Recently it is shown that a layer of force-free curent surrounding the main plasma core can lead to large increases in the maximum possible $\beta$ (or critical $\beta$ ) in a tokamak configuration. We will survey, high $\beta$ tokamakswith a layer of force-wree current from the point of stability to external kink modes.

Table I shows the main references according to our classification. In the following we will give brief explanations and survey MHD stability of external kink modes, noting the stability criterion, growth rates and critical $\beta$ of high B tokamaks.

\section{2-1 Low $\beta$ Tokamaks}

$A$ low $\beta$ tokamak means an axisymmetric toroidal plasma with $\beta_{p} \sim^{1}, \beta \sim \varepsilon^{2}$ and ${ }_{p}{ }_{p} B_{T}{ }^{2} \varepsilon$, implying that containment is basically achieved by the poloidal field ${ }_{\mathrm{p}}$. Most tokamak experiments are carried out in this regime. 


\section{2-1-1 Circular Cross-Section Tokamak}

Firstly we consider a cylindrical plasma column of radius a and periodic length $L=2 \pi R$, imbedded in a vacuum'with a longitudinal field $B_{z}$. Inside the cylinder we have a uniform plasma with

$$
P=P_{0}, \rho=\rho_{0}, B_{\theta}=0, B_{z}=B_{i}, J_{\theta}=J_{z}=0
$$

Outside the plasma column the magnetic fields are

$$
B_{\theta}=B_{p} a / r, \quad B_{z}=B_{0}
$$

Only in the surface $r=a$ we have

$$
j_{\theta}=j_{\theta a}=\left(B_{i}-B_{0}\right) / 4 \pi, j_{z}=j_{z a}=B_{p} / 4 \pi
$$

The equilibrium is defined by

$$
\mathrm{B}_{\mathrm{p}}^{2}+\mathrm{B}_{0}^{2}=\mathrm{B}_{\dot{\mathrm{i}}}^{2}+8 \pi \mathrm{P}_{0}
$$

The stability to external kink modes is investigated by use of linearized MHD equations. Since the geometry is simple, the linearized MHD equations can be solved easily. The solution has a form in which a dependent variable is a function of $r$ only multiplied by exp $(\omega t+i m \theta+i k z)$, where $\omega, m$ and $k$ are constants and a poloidal mode number $m$ is integer and $k$ is real. By use of the known boundary conditions, ${ }^{3}$ ) the dispersion relation for kink modes,

$$
4 \pi \rho_{0} \omega^{2}=-B_{i}^{2} k^{2}+\left(\frac{m_{B}}{a} p+k B_{0}\right)^{2} \frac{I_{m}^{\prime}(k a) K_{m}(k a)}{I_{m}(k a) K_{m}(k a)}+\frac{k B_{p}^{2}}{a} \frac{I_{m}^{\prime}(k a)}{I_{m}(k a)}
$$


is obtained for the above skin current equillibrium without a conducting wall. For the low $\beta$ tokamak, $B_{i}=B_{0}=B_{z}, B_{z}>B_{p}$ and $k a<<1$, eq. (5) assumes the simpler form

$$
4 \pi \rho_{0} \omega^{2}=-k^{2} B_{z}^{2}-\left(k B_{z}+\frac{m}{a} B_{p}\right)^{2}+\frac{m}{a} B_{p}^{2},
$$

and $\omega_{\max }^{2}$ can be found

$$
\omega_{\max }^{2}=\frac{B_{p}^{2}}{4 \pi \rho_{0} a^{2}}\left(m-\frac{m^{2}}{2}\right) .
$$

Only the $m=1$ mode grows in time and the cylindrical tokamak plasma is stable against the remaining modes with $m \geq 2$. This means an infinitely long cylindrical tokamak is unstable only against the $\mathrm{m}=1 \mathrm{kink}$ mode. However for a cylindrical tokamak of finite length, $k$ cannot be smaller than $2 \pi / \mathrm{L}$. It is evident from (6) that $\omega^{2}$ is positive for $|k|<^{B} \cdot p^{3} / a_{z}$; consequently a low $\beta$ tokamak of finite length is stable if

$$
\frac{B_{p}}{B_{z}}<\frac{2 \pi a}{L} \text { or } q \equiv \frac{a}{R} \frac{B_{z}}{B_{p}}>1
$$

This Kruskal1) - Shafranov ${ }^{2)}$ condition is a necessary condition for the stability of a low $\beta$ tokamak.

Shafranov extended the stability analysis of kink modes to a cylindrical tokamak of uniform plasma current and diffuse current profile. ${ }^{4}$ For a low $\beta$ tokamak of uniform current, 


$$
\begin{array}{lr}
\overrightarrow{\mathrm{B}}=\left(0, \mathrm{~B}_{\mathrm{p}} \mathrm{r} / \mathrm{a}, \mathrm{B}_{z}\right) & \text { for } r \leq \mathrm{a} \\
\overrightarrow{\mathrm{B}}=\left(0, \mathrm{~B}_{\mathrm{p}} \mathrm{a} / \mathrm{r}, \mathrm{B}_{\mathrm{z}}\right) & \mathrm{a}<\mathrm{r}<\mathrm{c}
\end{array}
$$

kink modes become unstable if

$$
m-I+\left(\frac{a}{c}\right)^{2 m}<n q<m
$$

is satisfied, where $c$ denotes the position of the conducting wall and a toroidal mode number $n$ is defined by $k=\frac{2 \pi}{L} n$. For $m=n=1$ mode, stability condition becomes the $k-s$ condition $q>1$. The growth rate of $(m, n)$ kink mode is given for $k a<<1$;

$$
w^{2}=\frac{\mathrm{B}_{\mathrm{p}}^{2}}{4 \pi \rho_{0} \mathrm{a}^{2}}\left[2(\mathrm{~m}-\mathrm{nq})-\frac{2}{1-\left(\frac{\mathrm{a}}{\mathrm{c}}\right)}(\mathrm{m}-\mathrm{nq})^{2}\right]
$$

and the maximum growth rate is

$$
\omega_{\text {max }}^{2}=\frac{B_{p}^{2}}{8 \pi \rho_{0} a^{2}}\left[1-\left(\frac{a_{c}^{2 m}}{c}\right)\right]
$$

Condition (10) shows the appearance of the stability window due to the presence of the conducting wall. The external kink modes are stabilized by the conducting wall for $c \grave{a}$. The growth rate of kink modes (11) is characterized by the transit time of poloidal Alfven velocity $v_{p A}=B_{p} / 4 \pi \rho_{0}$

For a low $B$ tokamak of diffuse current profile, Shafranov obtained important results that kink modes with $m \geq 2$ are stabilized if a current density is sufficiently concentrated in the center of a plasma column; however 
for the $m=1$ external kink mode the $K-S$ condition $q(a)>1$ is not changed. Shafranov also pointed out that the $m=1$ internal. kink mode becomes unstable in the cental region if $q(0)<1$ for a peaked current profile (which will be surveyed in \$3). The $m=1$ internal kink mode has a smaller growth rate than the external kink mode in a circular cross section tokamak. Low $\beta$ tokamaks are stable against external kink modes for $q(a) \succsim 3 \sim 4$ may be explained by Shafranov's stability analyses in cylindrical approximation.

Next we move to the stability analyses of external kink modes in a toroidal low $\beta$ tokamak. For a low $\beta$ tokamak of uniform current, Frieman et al. ${ }^{5)}$ studied MHD stability of external kink modes analytically in a torus of large aspect ratio $R / a>>1$. In order to investigate the effect of toroidal curvature on kink modes in tokamak configurations, stäbility criteria are derived by means of an expansion in the inverse aspect ration $\varepsilon=a / R$. For a low $\beta$ tokamak, $\beta_{p} \sim 1$ and $\beta \sim \varepsilon^{2}$, with a nearly constant safety factor, the stability criterion (10) is determined in second order of $\varepsilon$. It is shown that toroidal effects modify this criterion favorably in 4 th order of $\varepsilon$ when the vacuum region does not extend too far. Improvement of stability due to magnetic well is achieved when $\mathrm{c} / \mathrm{a}$ is less than $1.25 \sim 1.5$ typically. The minimum value of $a / c$ for stability is given;

$$
\left(\frac{a}{c}\right)^{6 m+6}>\frac{27 \beta^{2} a^{2} m^{2}}{4 R^{2}(m+1)(m+2)}
$$


where $\beta=\beta p \varepsilon^{2} / q^{2}$, When the wall is far from the plasma, the effect of the magnetic well is overwhelmed by balooning effects which deteriorate stability. Here it is noted that curvature effects tend to be destabilizing for a/c small and stabilizing for a/c large. This result is confirmed by several numerical studies of MHD stability in the toroidal geometry. Grimm and Johnson") use an analytic equilibrium with constant $p^{\prime}(\psi)$ and $I^{2}(\psi)$, such that

$$
\psi \propto R^{2} z^{2}+\frac{\alpha^{2}}{4}\left(R^{2}-R_{0}^{2}\right)^{2}
$$

where $(R, \phi, z)$ denote cylindrical coordinates and $R_{0}$ means the magnetic axis. This model has a nearly constant toroidal current density but a cross-sectional shape that can be adjusted to represent a non-circular cross section by $\alpha$, which gives the ratio of half axes. For $\varepsilon=1 / 6, \alpha=1$ (nearly circular cross-section) and $n=1$, a normalized growth rate for external kink modes is shown in Fig. 1. The growth rates are normalized to the poloidal velocity at the magnetic axis. Fig. 2 shows external kink modes are always unstaile for a low $\beta$ tokamak of uniform current, which is consistent with Ref. 5. The maximum growth rate is nearly equal to (12), which gives $-\Omega^{2}=0.5$. Berger et all) choose the following equilibrium

$$
\psi(R, z)=\frac{E}{2 q_{0}} \frac{a^{2}}{R^{2}}\left[\frac{R^{2} z^{2}}{E^{2}}+\frac{\left(R^{2}-K^{2}\right)^{2}}{4}\right]
$$

where E denotes a parameter for non-circularity, $q_{0}=q(0)$. 
If $\mathrm{E}=1$, the equilibrium is the same as the circular cross-section equilibrium given by (14). For a small aspect ratio $\mathrm{R} / \mathrm{a}=3$ and $\mathrm{c}=\infty$, normalized growth rates are shown in Fig. 2. Note that for: $R / a=3$ there is already a substantial amount of shear and these parameters give $q(a) / q(0)=1.74$. This may explain the reduction of growth rates compared with Fig. I. A conducting wall effect is shown in Fig. $3^{13)}$ for $\varepsilon=1 / 6, c / a=2$ and $\alpha=1$ (see solid curve). The conducting wall has an effect to reduce growth rate compared to Fig. 1 .

There is another competing effect on external kink modes which should be considered in a toroidal geometry: That is a current profile differing from the uniform current. Stability analysis of external kink modes in a low $\beta$ toroidal plasma with a diffuse current profile remains to be done in future studies. Skin current equilibrium with $\beta_{p}=1$ is investigated in the calculation of critical $\beta$ for a sharp boundary high $\beta$ tokamak by Freidberg and Grossmann ${ }^{16}$ ) (see $\$ 2-2-1$ ). 2-1-2 Non-Circular Cross-Section Tokamak

First we'will consider an elliptical cross-section. Stability for external kink modes in the elliptical straight tokamak with surface current is given by Laval and Pellat.) and Coppi et al. ${ }^{9}$ (hey treated small ellipticity limit and obtained marginal values of $q$ for the first two 
unstable kink modes,

$$
\begin{aligned}
& q=1+\frac{9}{8}\left(\frac{b-a}{b+a}\right)^{2} \text { for } m=1 \\
& q=1+\left(\frac{3}{2}\right)^{\frac{1}{2}} \frac{b-a}{b+a} \text { for } m=2
\end{aligned}
$$

where $\mathrm{b}$ and $\mathrm{a}$ denote major axis and minor axis of ellipse, respectively. Here $q$ is defined by

$$
\mathrm{q}=\frac{\mathrm{B}_{\mathrm{z}}}{2 \pi \mathrm{R}} \frac{\mathrm{d} \ell}{\mathrm{B}_{\mathrm{p}}}
$$

where di means a line element of elliptic cross-section. For slightly elliptical cross-sections with a skin current, (16) shows that the value of $q$ for stability increases with ellipticity. These conditions, as a limiting case, give $q=1$ for a circular cross section.

Next we consider an infinitely long cylindrical ${ }^{11}$ ) plasma of elliptical cross-section confined by an axial field $B_{z}$ with a uniform axial current $J_{z}$ inside the plasma. In the low $\beta$ tokamak the axial field $B_{z}$ can be treated as a constant $B_{0}$ and the poloidal field $B_{p}$ described by a flux function $\psi(x, y)$ such that

$$
\begin{aligned}
& \overrightarrow{\mathrm{B}}_{\mathrm{p}}=\hat{\mathrm{e}}_{\mathrm{z}} \times \nabla \psi \\
& \nabla^{2} \psi=\mathrm{J}_{\mathrm{z}}
\end{aligned}
$$

Inside the plasma

$$
\left.\psi=\psi_{0} \frac{\left(\underline{x}^{2}\right.}{a^{2}}+\frac{y^{2}}{b^{2}}\right)
$$


where $\psi_{0}=J_{z} a^{2} \cdot b^{2} / 2\left(a^{2}+b^{2}\right)$ is the total poloidal flux inside the plasma column, For the elliptic tokamak with a uniform current the magnetic surfaces within the plasma are similar ellipses. In this case the safety factor is given by

$$
q=\frac{2 \pi\left(a^{2}+b^{2}\right) B_{0}}{L a b J_{z}}
$$

Laval et $a 1^{10}$ ) and Dewar et al.11) investigated MHD stability of external kink modes by means of the energy principle. For an elliptic equillibrium with a uniform current, the range of instability of external kink mode with $(m, n)$ is given by

$$
\begin{aligned}
& \left(m-\frac{1+k^{2}}{1-k^{2}}\left(1-\alpha^{2 m}\right) \frac{1+k^{m}}{1+k^{m} \alpha^{2 m}}\right)\left(m-\frac{1+k^{2}}{1-k^{2}} \frac{\left(1-\alpha^{2 m}\right)\left(1-k^{m}\right)}{1-k^{m} \alpha^{2 m}}\right) \\
& <n^{2} q^{2}<m^{2}
\end{aligned}
$$

where $k=(b-a) /(a+b)$ and $\alpha$ shows the parameter giving the conducting wall:

$$
\left\{\begin{array}{l}
\frac{x^{2}}{a^{\prime^{2}}}+\frac{y^{2}}{b^{\prime^{2}}}=1 \\
a^{\prime}=\frac{a+b}{2 \alpha}+\alpha \frac{(a-b)}{2} \\
b^{\prime}=\frac{a+b}{2 \alpha}-\alpha \frac{(a-b)}{2} \\
0 \leq \alpha \leq 1 .
\end{array}\right.
$$


We can recover (10) for a circular cross-section with a uniform current when $\mathrm{k}=0$. When $\mathrm{k} \neq 0, \mathrm{Fig}, 4$ shows the range of unstable $q$ for $\left(b^{\prime}-b\right) / a=0.3$. In this figure, the ratio $\left(b^{\prime}-b\right)$ is the minimum distance from the plasma vacuum interface to the wall. It is noted that the conducting wall defined by (23) does not exist on the flux surface. In Fig. $5 j_{0}$ is the current density which would correspond to $q=1$ for a circular cross-section tokamak with radius a. Then $\bar{j} / j_{0}$ is related to $q$ by

$$
\bar{j} / j_{0}=\frac{\left(1+k^{2}\right)}{\left(1-k^{2}\right)} q
$$

for a uniform current equilibrium. The left figure in Fig. 5 is given by (22) and (24). The right figure shows the case of bell-shaped current density profile. Here $\bar{j}$ is the average current density. Fig. 5 shows that high $m$ kink modes $(m \geq 2)$ can be stabilized by the concentration of current density in the center of the plasma column even in the elliptical cross-section tokamak. In Fig. 4 the shrinkage of the stable zone is apparent but the current density in these zones increases with k. Here we should note that the stability criterion for $m=1$ and $n=1$ kink mode is $q>1$, which is l.consistent with (16) for a skin current case. Another novel property found by Dewar et al.11) is the coupling between the kink modes and the Alfven waves. For the elliptic equilibrium of uniform current without the conducting wall, the growth 
rates of external kink modes are shown in Fig. 6 and Fig. 7. These figures show that external kink modes are always unstable and their growth rates are larger than those in the circular cross section tokamak of uniform current. It is seen that each kink mode is coupled by finite ellipticity to Alfven waves of lower $\mathrm{m}$. Since the dispersion curves lie below the curves of kink modes and Alfven modes for the circular case, the effect of the coupling is destabilizing, despite the fact that the Alfven waves are stable modes. Numerical studies in this configuration show that the growth rates of external kink modes are sensitive to the shape of the plasma density distribution. The growth rate in Fig. 6 and Fig. 7 is normalized with the poloidal Alfven velocity at the center of ellipse.

In the toroidal geometry, Berger et al. ${ }^{7}$ calculated growth rates of external kink modes by use of the uniform current equilibrium (10). Fig. 8 shows growth rate of $n=1$ kink mode for $R / a=3$ (a small aspect of ratio torus) and $E=2$. Comparing with the circular cross-section case (Fig. 2), there is a strong destabilization of the $\mathrm{m}=\mathrm{I}$ mode and a stabilizing trend in the high $q_{0}$ region (this may be due to shear since $q(a) / q(0)=1.74)$. Kerner ${ }^{3}$ ) obtained the growth rate with the same trend using the equilibrium (14) for $R / a=4$ and $\alpha=4$ (see Fig. 9). However for the case of $R / a=6$ 
and $\alpha=4$, the growth rate becomes larger than the circular cross-section equilibrium $(\alpha=1)$ and ellipticity destabilizes external kink modes (see Fig. 3). This is consistent with the analyses by means of cylindrical approximation. It should be noted that the modes with different m values are strongly coupled in the toroidal geometry.

For the JET (Joint European Tokamak) configuration, Wesson and Sykes ${ }^{14}$ ) calculated growth rates of $n=1$ external kink mode using a uniform current equilibrium. The JET has an aspect ratio 2.5 and the height/width ratio is 1.6 . In Fig. 10 dips in the growth rate curve seen in Fig. 8 or Fig. 9 disappear (this may be due to a very small aspect ratio)

For non-circular low B tokamaks, MHD stability analyses of external kink modes for general diffuse current equilibrium in the toroidal geometry also remain to be done for future studies. Skin current equilibrium with $\beta_{p}=1$ is treated in the calculation of critical $\beta$ for a sharp boundary high $\beta$ tokamak by Freidberg and Grossman ${ }^{16}$ ) (see §2-2-1). 


\section{$\underline{2-2}$ High $B$ Tokamaks}

Up to now we considered low $\beta$ tokamaks characterized by the ordering $\beta_{p} \sim 1, \beta \sim \varepsilon^{2}$. We now consider high $\beta$ tokamaks with the so-called high $\beta$ ordering ${ }_{{ }_{p}}^{\beta} \sim \varepsilon^{-1}, \beta \sim \varepsilon$. In this regime the most important instability is a kinkballooning mode. Here we specialize MHD stability to kink-balooning modes and critical $\beta$ determined by the stability analyses.

2-2-1 High $B$ Tokamaks and Skin Current

The first MHD stability study of high $\beta$ tokamak ${ }^{15)}$ was about the equilibrium of a sharp boundary and axisymmetric tokamak with uniform plasma pressure confined by currents flowing only in the plasma surface. The cross-section is assumed perfectly circular. After that the stability analyses were extended to non-circular cross-section tokamak by use of high $\beta$ ordering. 17)-19) Recently Freidberg and Grossman ${ }^{16)}$ gave the analysis valid for arbitrary $\beta$, arbitrary aspect ratio and arbitrary cross section. Because no approximations are made, their calculation helps sort out where the simpler theories are valid and gives actual unscaled predictions of critical $\beta$ for a variety of tokamak cross sections for the fully toroidal case.

The plasma cross-section of a toroidal plasma with a skin current is given by

$$
r=a g(\theta)
$$


The function $g(\theta)$ represents the cross-section of the plasma and is arbitrary. It is normalized so that $g(0)=g(\pi)=1$. In the sharp boundary constant pressure model the plasma has only a toroidal field. On the plasma surface this field is given by

$$
\mathrm{B}_{\phi}=\mathrm{B}_{i} /[1+\varepsilon g(\theta) \cos \theta]
$$

where $B_{i}$ is the internal toroidal field at midplane $\theta=\pi / 2$. In the vacuum region there are both a toroidal field and a poloidal field induced by toroidal currents flowing on the plasma surface. The toroidal field on the plasm surface can be expressed as

$$
\hat{\mathrm{B}}_{\phi}=\mathrm{B}_{0} /[I+\varepsilon \mathrm{g}(\theta) \cos \theta] \text {, }
$$

where $\mathrm{B}_{0}$ is the external toroidal field at $\theta=\pi / 2$. The normal component of the magnetic field on the plasma surface vanishes since the plasma surface is a flux surface. At the plasma surface, the poloidal field $\hat{B}_{p}(\theta)$ is determined from pressure balance across the plasma vacuum surface,

$$
\frac{\hat{B}_{p}^{2}(\theta)}{B_{0}^{2}}=\frac{8 \pi P_{0}}{B_{0}^{2}}-\frac{\left(1-B_{i}^{2} / B_{0}^{2}\right)}{[1+\varepsilon g(\theta) \cos \theta]^{2}}
$$

To specify an equilibrium it is necessary to give a shape 
factor $g(\theta)$ and a value for each of the three constants $\varepsilon, B_{1}^{2} / B_{0}^{2}$ and $8 \pi \mathrm{P} \alpha / B_{0}^{2}$ ( $O r, \varepsilon, \beta$ and $q$ ). On the plasma surface the safety factor is defined by

$$
q=\frac{1}{2^{\prime} \|} \int_{0}^{2 \pi} \frac{\varepsilon^{B} 0}{\widehat{B}_{p}} \frac{g(\theta) Q(\theta) d \theta}{T(i+\varepsilon g(\theta) \cos \theta)^{2}}
$$

Where $Q(\theta)=\left[1+\left(g^{\prime} / g\right)^{2}\right]^{\frac{1}{2}}$ and the prime denotes differentiation with respect to $\theta$. The plasma $\beta$ is also defined as a surface average;

$$
B=\frac{8 \pi\langle p\rangle}{\left\langle\hat{B}^{2}\right\rangle}=\frac{8 \pi P_{0} / B_{0}^{2}}{8 \pi P_{0} / B_{0}^{2}+\alpha B_{i}^{2} / B_{0}^{2}},
$$

where

$$
\begin{aligned}
\alpha= & \int_{0}^{2 \pi} g(\theta) Q(\theta)(1+\varepsilon g(\theta) \cos \theta)^{-1} d \theta / \\
& \int_{0}^{2 \pi} g(\theta) Q(\theta)(1+\varepsilon \cos \theta) d \theta .
\end{aligned}
$$

The poloidal $\beta$ is found by

$$
\beta_{p}=\frac{\beta_{B_{i}^{2} / B_{0}^{2}}}{\beta-1+B_{i}^{2} / B_{0}^{2}}
$$

Here we consider two limits. The first is the low $\beta$ limit where all the plasma pressure is confined by the poloidal field. In this case $B_{i}=B_{0}$ and eq. (31) gives $\beta_{p}=1$. Tinus from the low $\beta$ limit we require $\beta_{p} \geq 1$ when $B_{i}^{2} / B_{0}^{2} \leq 1$. The second limit is a high $\beta$ limit. As $B_{i}^{2} / B_{0}^{2}$ decreases from unity, more and more pressure is contained. Eventually, 
a second magnetic axis appears on the inner surface of the plasma. At this point $\hat{\mathrm{B}}_{\mathrm{p}}(\pi)=0$ and $q=\infty$. This is the socalled high $\beta$ tokamak regime. ${ }^{15)}$. From eq. (28) the high $B$ limit requires $q<\infty$ and

$$
B_{i .}^{2} / B_{0}^{2} \geq 1-(1-\varepsilon)^{2} 8 \pi P_{0} / B_{0}^{2}
$$

The stability analyses of the above equilibrium have been done by the energy principle. To calculate the critical $\beta$ for stability for a given shape $g(\theta)$, inverse aspect ratio $\varepsilon$, and safety factor $q$, Freidberg and Grossmann used numerical minimization of the potential energy $\delta w .{ }^{16)}$

For a circular cross-section described by

$$
g(\theta)=1
$$

the results of a computation are shown in Fig. 11. As in the high $\beta$ ordering the critical $\beta$ for stability levels off as $q \rightarrow \infty$. Above this value of $\beta$ the plasma is unstable to kink-ballooning modes for any $q$. As q decreases, the critical $\beta$ also decreases and eventually $\beta_{p}=1$ is reached. As the aspect ratio decreases the critical $B$ increases. Fig. 12 shows the critical $\beta$ at the limiting cases $q=\infty$ and $\beta_{p}=1$ for the circle. In the low $\beta$ ordering, $\beta_{p}{ }_{1}$, $\beta \sim \varepsilon^{2}$, the critical $\beta$ is given by $\beta=a^{2} / R^{2}$ when $\beta_{p}=1$ (see the conventional ordering line). In the high $\beta$ ordering, $\beta_{\mathrm{p}}{ }^{\tau^{-1}}$, $\beta \sim \varepsilon$, the critical $\beta$ is given by $\beta=0.21$ a/R when $q \rightarrow \infty$ ) (see the high $\beta$ ordering line). If $a, R>0.21$, the low $B$ ordering result 
becomes inconsistent, predicting a higher critical $\beta$ than from the high $B$ ordering. The numerical results indicate more pessimistic values of critical $B$ than the corresponding theoretical values, especially for the $\beta_{\mathrm{p}}=1$ case. Notice that the gain in critical $\beta$ in going from $\beta p=1$ to $q=\infty$ is quite small for $a / R>\frac{1}{4}$. This can be understood as follows: we might expect on the basis of the conventional tokamak expansion that when $\beta_{p}=1$, the value of $q$ for stability is $q=1$ (the KruskalShafranov limit). However, it turns out that for finite $a / R$, the critical $q$ is not unity, but an increasing function of $a / R$, even when $\beta_{p}=1$ (low $\beta$ ordering). This is shown in Fig. 13 .

Some insight into the nature of the unstable mode is obtained by examining the structure of the eigenfunction. Fig. (14) shows the relative harmonic content of the eigenfunction versus $q$ along the $n=1$ marginal stability curve. The marginal mode turns out to be predominantly $\mathrm{m}=2^{15}$ ) For $\mathrm{q} \approx 1$, the eigenfunction is predominanatly $m=2$ and $m=1$. As $q$ increases, the $m=3$ influence also increases. In low $\beta$ tokamak, the $\mathrm{m}=1$ is to be the worst mode.

For the elliptic cross-section tokamak,

$$
g(\theta)=\left[\cos ^{2} \theta+\left(\frac{a}{b}\right)^{2} \sin ^{2} \theta\right]^{-\frac{2}{2}}
$$

critical $\beta$ versus elongation is shown in Fig. 15 for the two limiting cases of equilibrium, $\beta_{p}=1$ and $q=\infty$. The curves indicate that for both $\beta_{p}=1$ and $q=\infty$ there is an optimum elongation for obtaining the maximum critical $\beta$. This elongation is about 2.2 for $q=\infty$ and 1.9 for $\beta_{p}=1$. A similar behavior was found in the high $\beta$ tokamak ordering 
analysis $^{17)}$ or in the cylindrical approximation, ${ }^{18}$ ) In Fig.16, the maximum critical $\beta$ is shown versus inverse aspect ratio for the two limiting cases $\beta_{p}=1$ and $q=\infty$. The corresponding result from the high $\beta$ ordering $\beta=0.37 \mathrm{a} / \mathrm{R}^{17}$ ) is shown as the dashed line. The quantitative features are very similar to the circular case. In order to investigate the relation between the toroidal calculation and the cylindrical approximation, Fig. 17 shows the variation of critical $q$ with $b / a$ in the limit $\beta / \varepsilon \rightarrow 0.17$ ) For either a vertical $(b / a>1)$ or horizontal ellipse $(b / a<1)$ the critical $q$ is a symmetric increasing function of ellipticity. This result is both qualitative and quantitative consistent with the cylindrical approximation for elliptic equilibrium with a skin current. $\left.{ }^{8}\right), 18$ ) The slope of the curve at $b / a=1$ is in agreement with the $m=2$ result (see (16)) of Laval and Pellat. ${ }^{8}$ ) The harmonic content (or m dependence) of the marginal stability curves depends very strongly on the particular value of b/a under consideration (see Fig. 18).17) In general, higher b/a contains high and more complicated mode structure. Freidberg and Grossman ${ }^{26}$ ) also calculated critical $\beta$ for the triangular cross-section tokamak and the square crosssection tokamak. In general, if the cross-section becomes triangular or square, without elongation, the critical $\beta$ is lower than for the circle. This is primarily due to concentrated regime of bad curvature near the corners. For the doublet configuration $g(\theta)$ is determined from 
numerically computed doublet equilibria. Doublets with different elongations are treated by simply scaling the vertical coordinate, leaving the horizontal coordinate unchanged. The stability results are shown in Fig. 19. The aspect ratio is fixed at $a / R=0.41$. It is seen that there is an optimum elongation for maximizing the critical 6. For $q=\infty$ this value is $b / a \simeq 4.1$ for $\beta_{p}=1, b / a \simeq 3.9$. The critical $\beta$ 's for the optimized doublet are very similar to those of the optimized ellipse.

For the race track configuration Marder ${ }^{19}$ ) calculated critical $b$ in the cylindrical approximation. The race track shape can be made much more elongated than the elliptic cross-section without affecting the stability.

The general features of non-circular cross-section tokamaks of skin current are as follows: Making the crosssection either triangular or square without elongation is destabilizing. Elongation itself can be helpful and for both the doublet and the ellipse there is an optimum elongation to obtain a maximum critical $\beta$.

The effect of wall stabilization on the $n=1$ kink mode is shown in Fig. 2015). The limiting value of critical $\beta / \varepsilon$ as $q \rightarrow \infty$ is shown vs a/c (plasma-wall ratio) for the $n=l$ mode. The conducting walls have a very small effect unless they are very close to the plasma.

The growth rate of unstable $n=1$ kink mode is shown in Fig. $21^{20)}$ for a circular cross-section equilibrium with a skin current and a large aspect ratio. The growth rate is 
normalized by a poloidal Alfven velocity and has nearly the same magnitude as the external kink mode in the low B tokamak. 
2-2-2 High $\beta$ Tokamak with Diffuse Current Profile

First we consider circular cross-section high $\beta$ tokamaks with nearly uniform toroidal current profiles. The MHD stability analysis of external kink modes in this configuration is an extension of Shafranov's kink mode analyses for $\beta \varepsilon^{2}{ }^{4}$ ) (see $(10)$ ) to $\beta \sim \varepsilon$.

The $\beta \sim E$ uniform current equilibrium has been given by . Haas. 2) The stability analysis consists of calculating the energy principle $\delta w$ to second order $\varepsilon^{2}$ and finding the condition for marginal stability. Since internal kink modes appear in $\varepsilon^{4}$, they are neglected in this analysis. The marginal stability results are presented as curves of the critical value of $\beta / n^{2} \varepsilon$ versus nq.

For the case of the conducting wall at infinity, instability for any $\mathrm{n}$ and all $\beta$ is found. This is consistent with the results of low $\beta$ tokamaks. Fig. 22 shows the marginal curve for $c / a=1.4 .^{21)}$ Taking $\beta \rightarrow \varepsilon^{2}$, the intercepts on the $\mathrm{nq}$ axis (corresponding to instability) agree with those derived from (10). However, unlike the low $\beta$ tokamak, the high $\beta$ toroidal model shows that the region of the stable windows depends on $\beta$ and $n$. With the wall at $c / a=1.2$ the stability windows have broadened (see Fig. 23). 21) With $c / a=1.1$ the broad stable area sweeps in and the diagram collapses to a series of small unstable regions (see Fig. 24).21) Finally, for the wall on the plasma case, $c / a=1$, the kink modes are completely stabilized, which is the same as the low $\beta$ tokamaks. Here we must bear in mind that the internal 
kink modes are neglected, From Fig, 22 - Fig. 24 the low $n$ kink modes can be stabilized by a choice of $q, \beta$ and the wall position.

Next we consider circular cross-section high $\beta$ tokamak with a diffuse rurrent profilc. Dy specifying the free function $p(\Psi)$ and $I^{2}(\Psi)$ in the Grad-Shafranov equation to be exponential, $e^{-\alpha \psi}$, Freidberg and Goedbloed ${ }^{22)}$ solved the following equation to leading order

$$
\Delta^{*} \psi=A(1+B r \cos \theta) e^{-\alpha \psi}
$$

where $A$ and $B$ are arbitrary constants related to $\beta$ and $q$. The main feature of the solutions of this equation is the fact that they centered around a magnetic axis which is not in the center of the circular conducting wall but shifted by an amount $\delta \delta$ a (a is assumed to be unity), where $\delta$ denotes a toroidal shift.

The stability analysis is carried out by means of the variational principle

$$
\omega^{2}=\delta w / \int \frac{1}{2} \rho \vec{\xi}^{2} d v
$$

and $\omega^{2}$ is calculated to order $\varepsilon^{2}$, which is the first nontrivial order in which both ballooning and kink terms are present when $\beta \sim \varepsilon$. Here $\delta w$ is the potential energy including the contributions of plasma, surface and vacuum.

The parameter a representing the freedom in choice of current and pressure profile is replaced by the parameter $a_{\text {eff }}=\sqrt{\langle\beta\rangle / \beta_{0}}$ (the width of the pressure and current profile). 
Here $\langle\beta\rangle$ is the average value of $\beta$ and $\beta_{0}$ is the value at the magnetic axis. Small. values of a eff imply a highly concentrated current profile with a lot of shear (large ratio of $q_{1} / q_{0}$ where $\left.q_{1}=q(a)\right)$, whereas large values of a eff refer to flat current profileswith little shear.

The results of the stability analysis are shown in Fig.25. Fig.25(a) gives the stability boundary for the very concentrated current profile with a wall radius $c / a=2$, There are two stable regions, one for small $\mathrm{nq}_{1}$ and another for $\mathrm{nq}_{1}>1$. In the region of small $\mathrm{nq}_{1}$, one can always find higher values of $\mathrm{n}$ for which the system is unstable. The region to the right of $\mathrm{nq}_{1}$ has physical significance. This region is in good qualitative agreement with the result obtained in ref. 15) for a high $\beta$ tokamak of a skin current for which a similar stable region was found for $\beta / \varepsilon<0.21$. Here $\langle\beta>/ \varepsilon<0.1$ is found, so that the skin current model is about a factor of two optimistic. Notice that the value of $q_{i}$ at the plasma surface is plotted. The situation of Fig. $25(a)$ corresponds to one where $q_{0}$ is much smaller than 1. In this case the internal kink modes become unstable, ${ }^{4}$. which are not depicted in Fig. $25(\mathrm{a})$, since the calculation has only been carried out up to second order of $\varepsilon$. In this order internal kink modes do not appear as is also the case for a low $\beta$ tokamak with a circular cross-section.

In the case of Fig. $25(\mathrm{~b})$ the shear is much smaller so that the stable region starts to show the tendency similar to the stability of a uniform current case. If the conducting wall is much more close to the plasma; the stable region 
starts to grow and even merge in the upper parts of the diagram (see Fig, 25(c) and Fig. 25(d)). This peculiar behavior, implying the possibility of stabilization by high $\beta$, is seen in Fig. 23 and Fig, 24 for the uniform current equilibrium.

It is shown that MHD stability of the $\omega^{2} \lambda \dot{\varepsilon}^{2}$ time scale: can be obtained by the conducting wall close to the plasma, $c / a \leqslant 1.2$. However it is unknown whether a wall-stabilized high $\beta$ tokamak can be realized experimentaliy or will the internal kink modes become crucial.

\section{2-2-3 High $\beta$ Tokamak with Force-Free Currents}

So far we considered the toroidal plasma column surrounded by a vacuum. The Jutphaas group pointed out that the external' kink modes are stabilized by the layer of force-free currents: Schuurman et al. 23) showed stable region for external kink modes with $q \sim 0.75$ and $B \sim 0.2$ for a skin current cylindrical plasma surrounded by a constant pitch force-free field. A region of constant pitch of the magnetic field lines, extending from the main plasma column to the conducting wall, turns out to be effective in suppressing the external kink modes. However, because of absence of shear in the outer region, it suffers from new unstable modes which are localized in the force-free region. Goeldbloed ${ }^{24}$ ) studied constant a force-free fields (when $\alpha$ is defined by $\vec{\nabla} \times \vec{B}=a \vec{B})$ and showed a skin current plasma surrounded by a pressureless plasma with force-free magnetic fields of constant $\alpha$ is stable to external kink modes with $\beta \sim 0.2$. Its property of having a high shear also suppresses localized modes. 
From another point of view, 'o-stable equilibrium ', Sakanaka and Goedbloed ${ }^{25}$ ) investigated cylindrical diffuse pinch surrounded by a layer of force-free current as one of the examples. The concept of $\sigma$ stability is as follows. For thermonuclear confinement plasma, it is not necessary to demand absolute MHD stability, but that it is sufficient that perturbations do not grow faster than exp $(\sigma t)$, where $1 / \sigma$ corresponds to some characteristic time needed for fusion. It is found that a diffuse pinch surrounded by a layer of force-free currents is ' $\sigma$ stable' for $\beta \sim 0.3$ but $j_{\theta}, j_{z} \neq 0$ at the conducting wall. When $j_{\theta}, j_{z}=0$ at the conducting wall, $\beta$ reduces to 0.08 .

In the cylinarical approximation, there is no work to investigate stability to external kink modes in non-circular cross-section tokamaks with a layer of force-free currents.

In 2-2-1, there has been a large effort aimed at maximizing $\beta$ in sharp boundary high $\beta$ tokamaks by appropriately shaping the cross-section. Recently D'Ippolito et al. 26) showed that a layer of force-free currents surrounding the main plasma column can lead to large increases in the maximum possible $\beta$ in high $\beta$ tokamak.

From the equilibrium point of view the addition of force-free currents outside the plasma column has little effect in the value of $q(0)$ which is determined by the internal current. The external force-free current should be able to support a higher total pressure by providing more flux to compress against the conducting wall. 
For investigation of MHD stability D'Ippolito et al use the skin current model. The effects of force-free currents are included by surrounding the main plasma with a region of pressureless plasma, in which $\vec{V} \times \vec{B}=\alpha \vec{B}$. 'line case of $\alpha=$ constant is treated. The force-free region is assumed to extend from the main plasma to a conducting wall. The stability studies have been carried out for a variety of cross-sections, including the circle, the ellipse and the race track. The point of view is that of exploring the relative gain in external kink mode stability as a function of elongation and amplitude of force-free current. The results for the circular cross-section are shown in Fig. 26. Here

$$
\Gamma=-\alpha \mathrm{Rq} *
$$

is the quantity which measures the amplitude of the forcefree currents and

$$
q^{*}=\frac{d^{2} B_{0}}{2 \pi \mu_{0} R I}
$$

where $I$ is the total toroidal skin current and $d$ is the circumference of the cross section. The curve has a maximum and at the optimum point $\Gamma=2, \beta / \varepsilon=0.62$ is found. The force-free currents increase the critical $\beta / \varepsilon$ by a factor of 3 over the vacuum case $\left(\beta / \varepsilon=0.21^{15}\right)$ ). The condition $\Gamma=2$ corresponds to approximately constant pitch in the equilibrium force-free fields. For the ellipse and the race track case, $\beta / \varepsilon$ versus elongation $b / a$ is shown in Fig. 27, Each point on the curve corresponds to 
the maximum $\beta / \varepsilon$ obtained by varying $\Gamma$. For the ellipse we see that there is an optimum elongation $b / a \approx 3.8$. At this point $\Gamma=4,0$ and the value of $B / E$ is given by $\beta / \varepsilon=1.17$. This should be compared with the elliptical high $\beta$ tokamak surrounded by vacuum, ${ }^{17)}$ where the optimum condition is $b / a=2.2$ and $\beta / \varepsilon=0,37$. We see that the force-free currents improve the maximum $\beta / \varepsilon$ for the ellipse by a factor of about 3. The race track is even more stable than the ellipse. The results indicate that at an elongation of 4 , the maximum $\beta$ is given by $\beta / \varepsilon=2.50$, representing a further increase of a factor 2 over the ellipse.

In the stability analyses presented above the effects of the conducting wall is ignored. For a circular cross-section high $\beta$ tokamak with a constant-pitch force-free field, stability analysis to external kink modes is carried out in the toroidal geometry of large aspect ratio, $a / R \ll 1^{27)}$. Here stabilization of external kink modes by a layer of force-free currents is shown by including the effects of the conducting wall. The result is much more stable than the sharp boundary high $\beta$ tokamak covered by the conducting wall.

The MHD stability analyses for realistic diffuse plasma surrounded by a layer of force-free currents in the toroidal geometry remain for future studies. A proper combination of elongation and force-free currents is required to obtain high value of $\beta$ in the tokamak configuration. 
References of Section 2

1. M.D. Kruskal, J.L. Johnson, M.B, Gottlieb and L.M. Goldman: Phys. Fluids 1,421 (1958).

2. V.D. Shafranov: Sov. Phys.- JETP G, 545 (1958).

3. B.B. Kadomtsev, in Reviews of Plasma Physics, edited by M.A. Leontovich (Consultant Bureau, New York,) vol. 2, p. 153.

4. V.D. Shafranov: Sov. Phys - Technical Physics 15, 175 (1970).

5. E.A. Frieman, J.M, Green, J.I. Johnson and K.E. Weinmer: Phys. Fluids 16, 1108 (1973).

6. R.C. Grimm and J.L. Johnson: Matt-1269 (Princeton University), 1976.

7. D. Berger, L.C. Bernard, R. Gruber and F. Troyon: 6th IAEA Conference on Plasma Physics and Controlled Nuclear Fusion Research (Berchtesgaden), (1976).

8. G. Laval and R. Pellat: EUR-CEA-575 (1970).

9. B. Coppi, R. Dagazian and R. Gajewski: Phys. Fluids 15,2405 (1972).

10. G. Laval, R. Pellat and J.S. Soule: Phys. Fluids 17, 835 (1974).

11. R.L. Dewar, R.C. Grimm, J.L. Johnson, E.A. Frieman, J.M. Green and P.H. Rutherford: Phys. Fluids 17, 930 (1974).

12. G. Laval and R. Pellat, in Proc. 3rd Int. Symp. on Toroidal Plasma Confinement, München, F2 (1973).

13. W. Kerner: Nuclear Fusion 16, 639 (1976).

14. J.A. Wesson and A. Sykes in Proc. 5th IAEA Conference on Plasma Physics and Controlled Nuclear Fusion Research (Tokyo), vol. I, p. 449 (1974).

15. J.P. Freidberg and F.A. Haas: Phys. Fluids 16, 1909 (1973). 
16. J.P. Freidberg and W, Grossmann: Phys, Fluids 18, 1494 (1975).

17. J.P. Freidberg and F.A, Haas: Phys. Fluids 17, 440 (1974).

18. B.M. Marder: Phys, Fluids 17, 447 (1974).

19. B.M. Marder: Phys. Fluids 17, 634 (1974).

20. M. Wakatani: J. Phys. Soc. Japan 38, 1481 (1975).

21. J.P. Freidberg, J.P. Goedbloed, W. Grossmann and F.A. Haas in Proc. 5th IAEA Conference on Plasma Physics and Controlled Nuclear Fusion Research (Tokyo), vol. I, p. 505 (1974).

22. J.P. Freidberg and J.P. Goebloed in Proc. 3rd Topical Conference on High $\beta$ Pulsed Plasmas (Culham) 1975.

23. W. Schurman, C. Bobeldjik and R.F. de Vries: Plasma Physics 11, 495 (1969).

24. J.P. Goedbloed: Phys. Rev. Lett. 24, 253 (1970).

25. P.H. Sakanaka and J.P. Goedbloed: Phys. Fluids 17, 919 (1974).

26. P.A. D'lppolito, J.P. Freidberg, J.P. Goedbloed and J. Ram: 6th IAEA Conference on Plasma Physics and Controlled Nuclear Fusion Research (Berchtesgaden), (1976) CN-35/E22.

27. M. Wakatani: J. Phys. Soc. Japan (to be published).

28. F.A. Haas: Phys. Fluids 15, 141 (1972). 


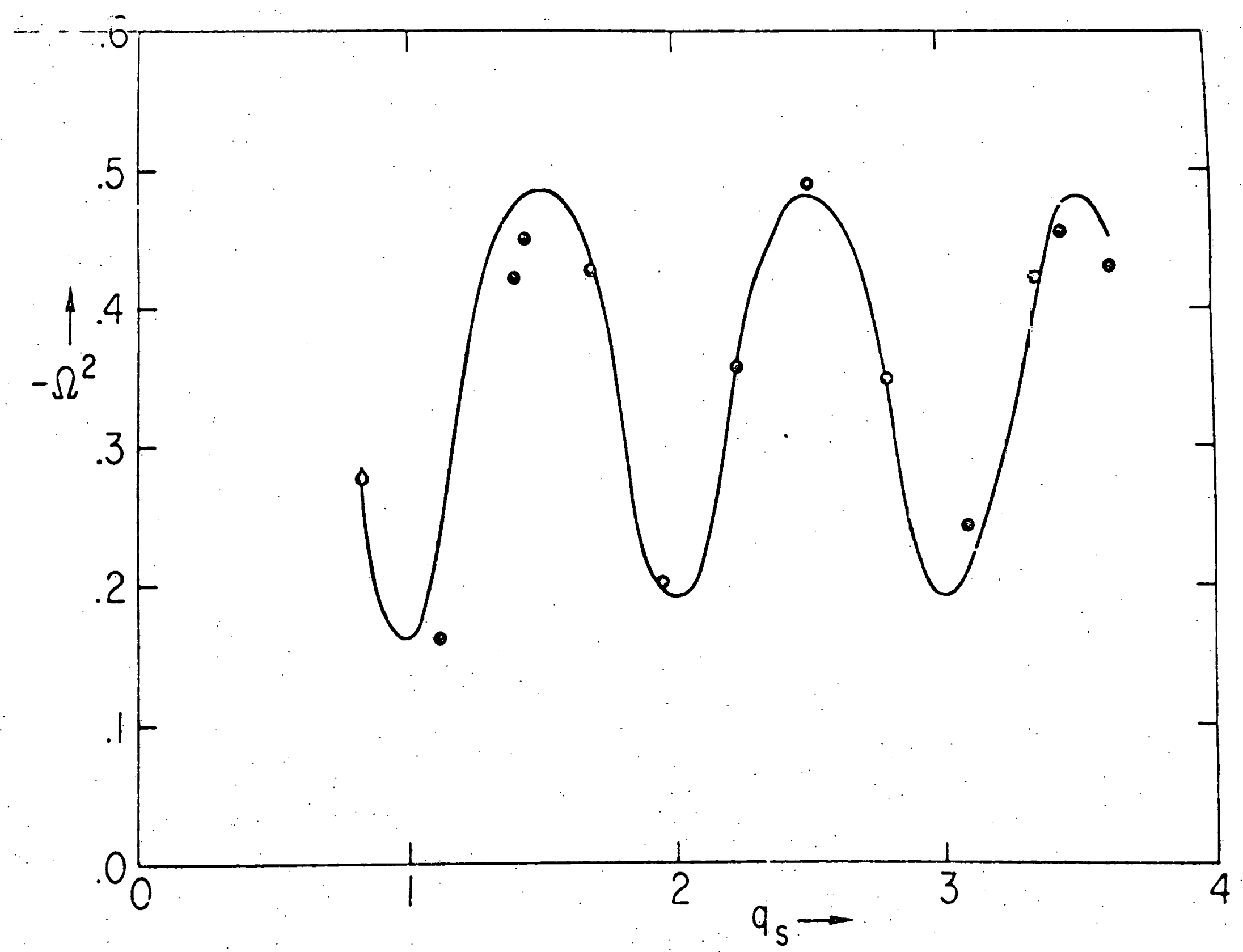

Fig. 1. The normalized growth rate of the most unstable mode for the analytic equilibrium (14) with $E=1 / 6$. (Ref. 6). 


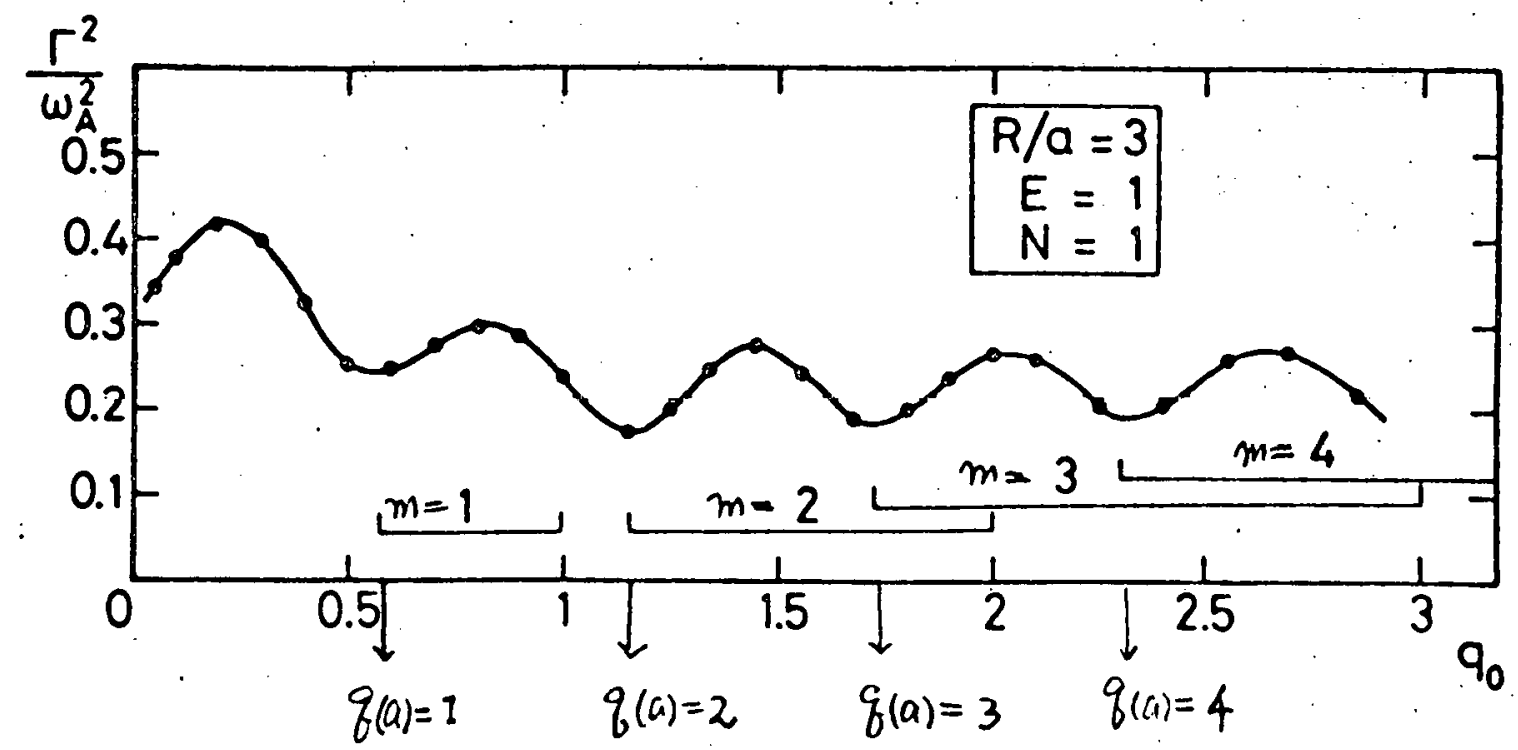

Fig. 2. The normalized growth rate of the most unstable mode for the equilibrium (15) with $\varepsilon=1 / 3, E=1$ (circular cross section) and $\mathrm{N}$ (torroidal mode number) $=1$ (Ref. 7).

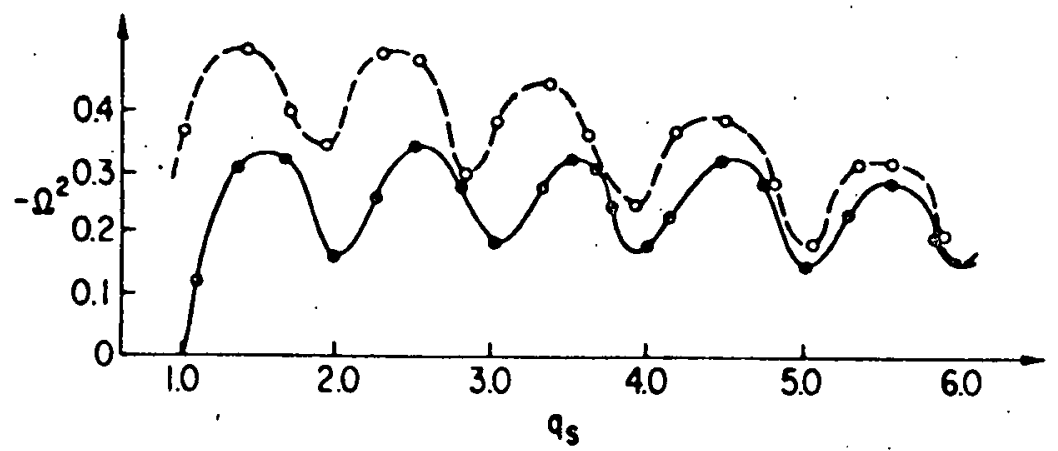

Fig. 3. The normalized growth rate for $\varepsilon=1 / 6, c / a=2$ and $\alpha=1$ (see solid curve). The dashed curve represents $\alpha=4$ (Ref. 13). 


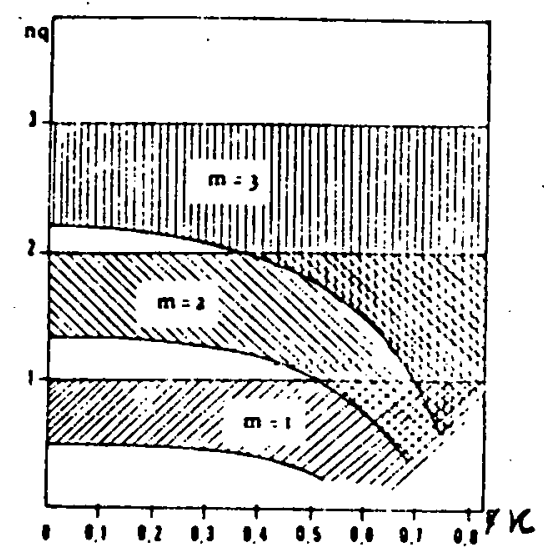

Fig. 4. Stability diagram on the plane nq,k; shaded areas 1 correspond to instability (Ref. 10).
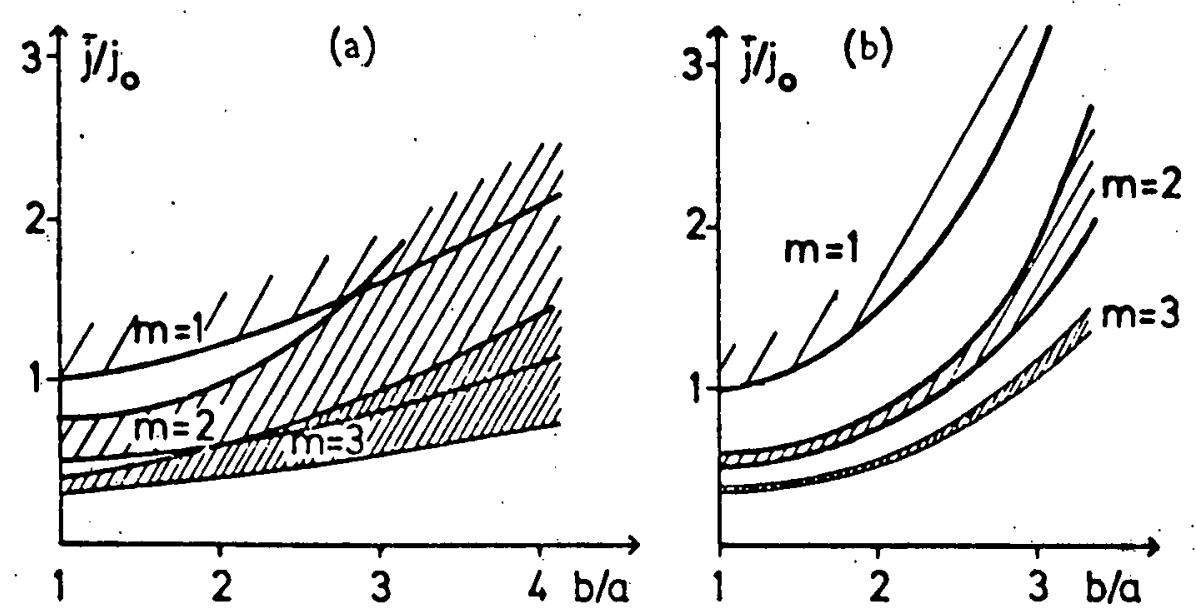

Fig. 5. Limits of the unstable domains for $m=1,2$ and 3 modes, respectively, as a function of $\mathrm{b} / \mathrm{a}$; (a) uniform current density profile, (b) bell-shaped current density profile (Ref. 12). 


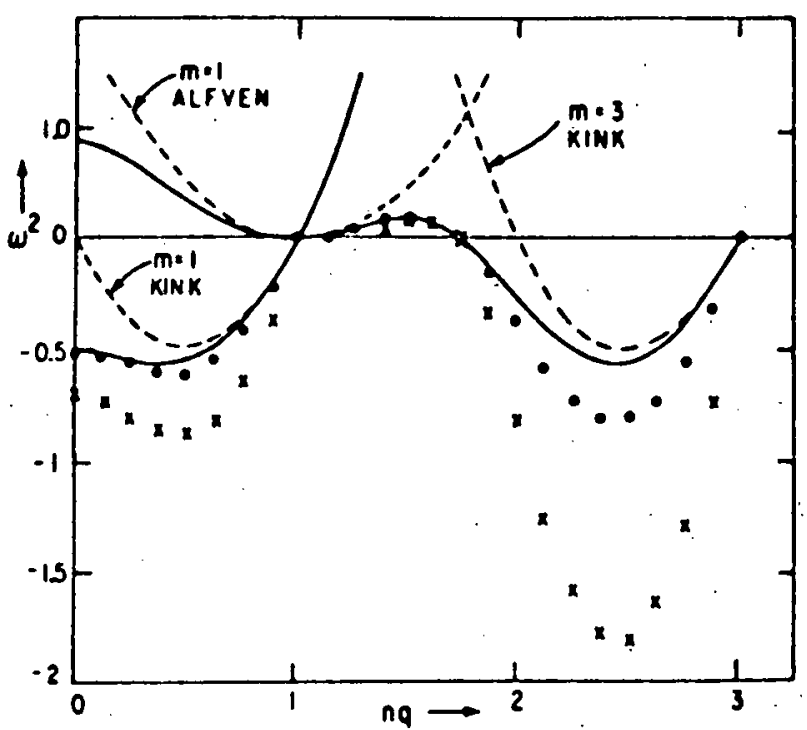

Fig. 6. The lowest branches of the $m=1$ and $m=3$ dispersion relations for the uniform density case for $a / b=1.0$ (dashed lines) and 0.5 (solid lines). Also shown are numerical results for $a / b=0.5$ with a parabolic density profile such that $\rho(1) / \rho(0)=0.25$ (dots) and a gaussian profile such that $\rho(1) / \rho(0)=0.044$ (crosses) (Ref: 11).

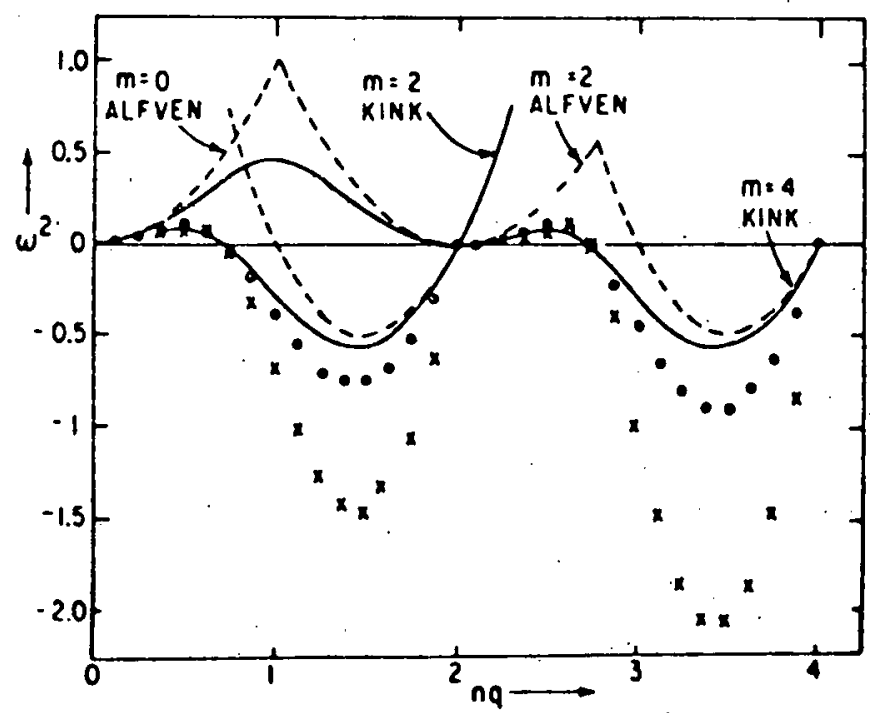

Fig. 7. The lowest branches of the $m=2$ and $m=4$ modes for the same cases as in Fig. 6. (Ref. 11). 


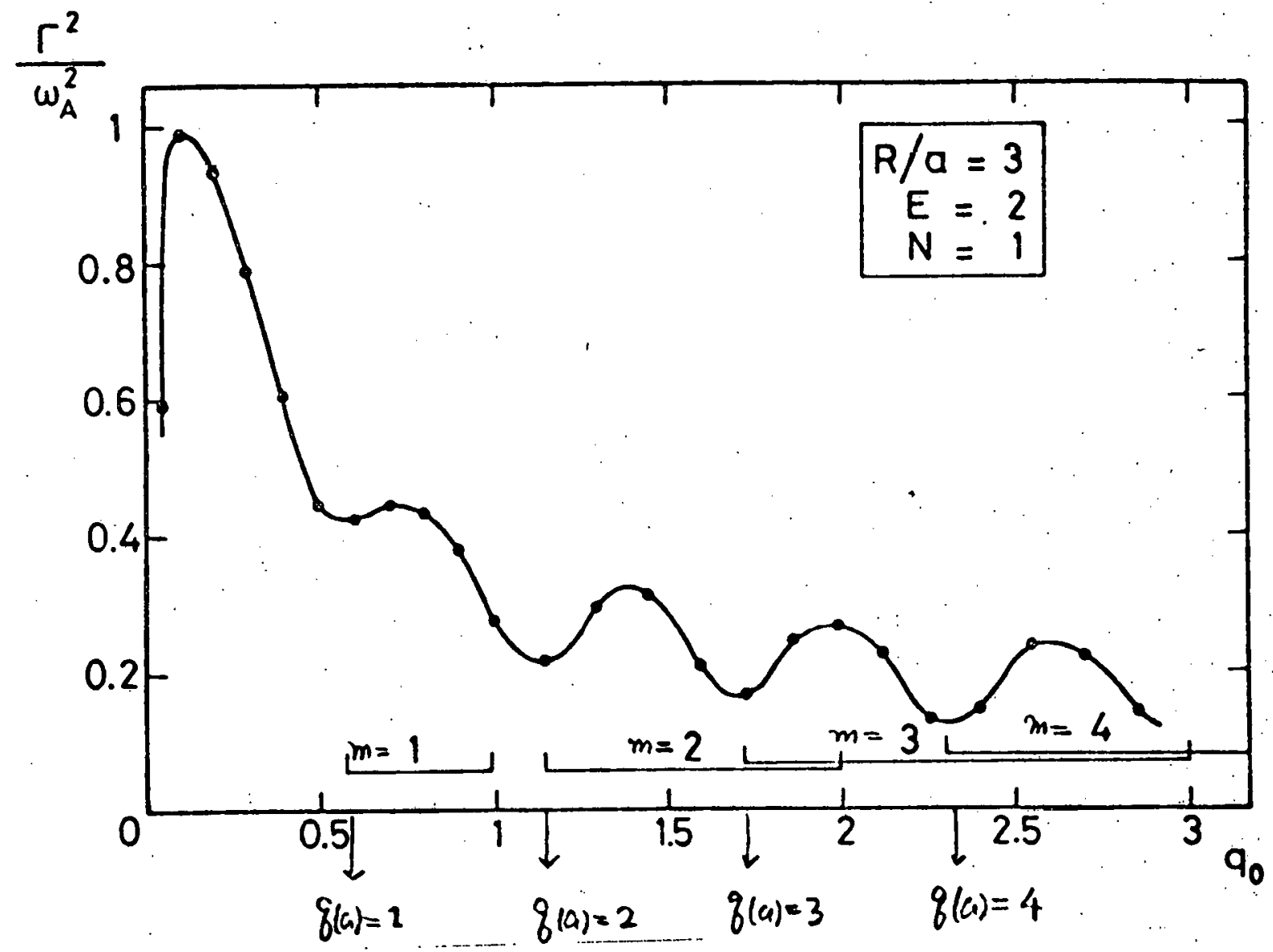

Fig. 8. Plot of the normalized growth rate of the $E=2, n=1$ kink mode versus $q_{0}=q(0)$ 
41

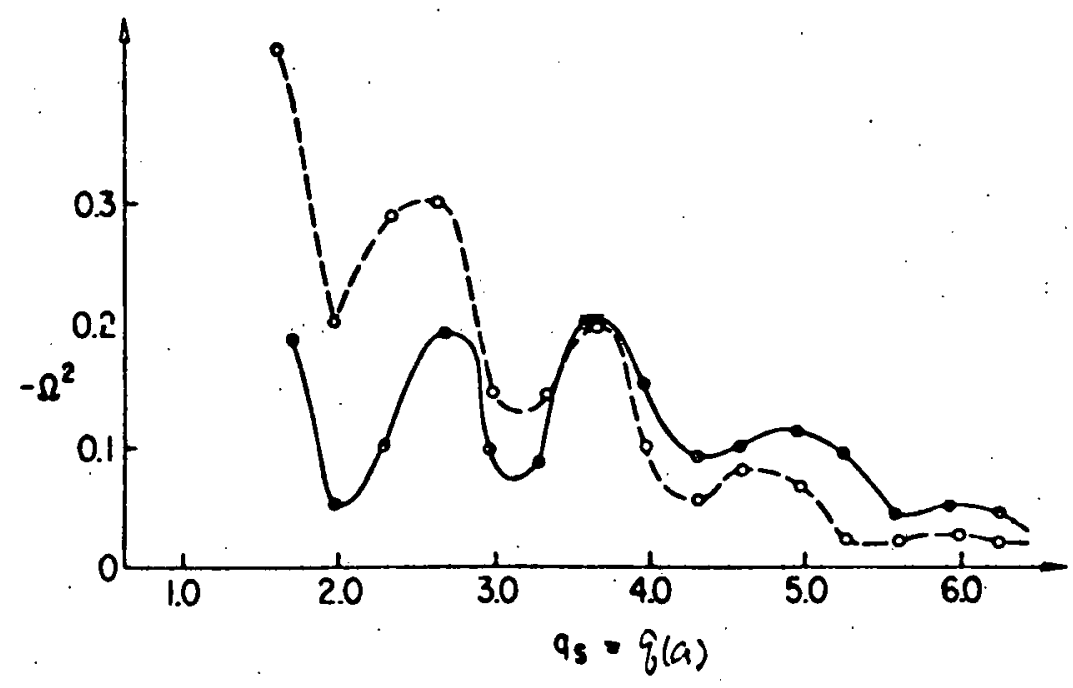

Fig. 9. The normalized growth rate for $\varepsilon=1 / 4, \cdots c / a=1.5$, The solid curve represents $\alpha=1$ and the dashed curve represents $\alpha=4$.

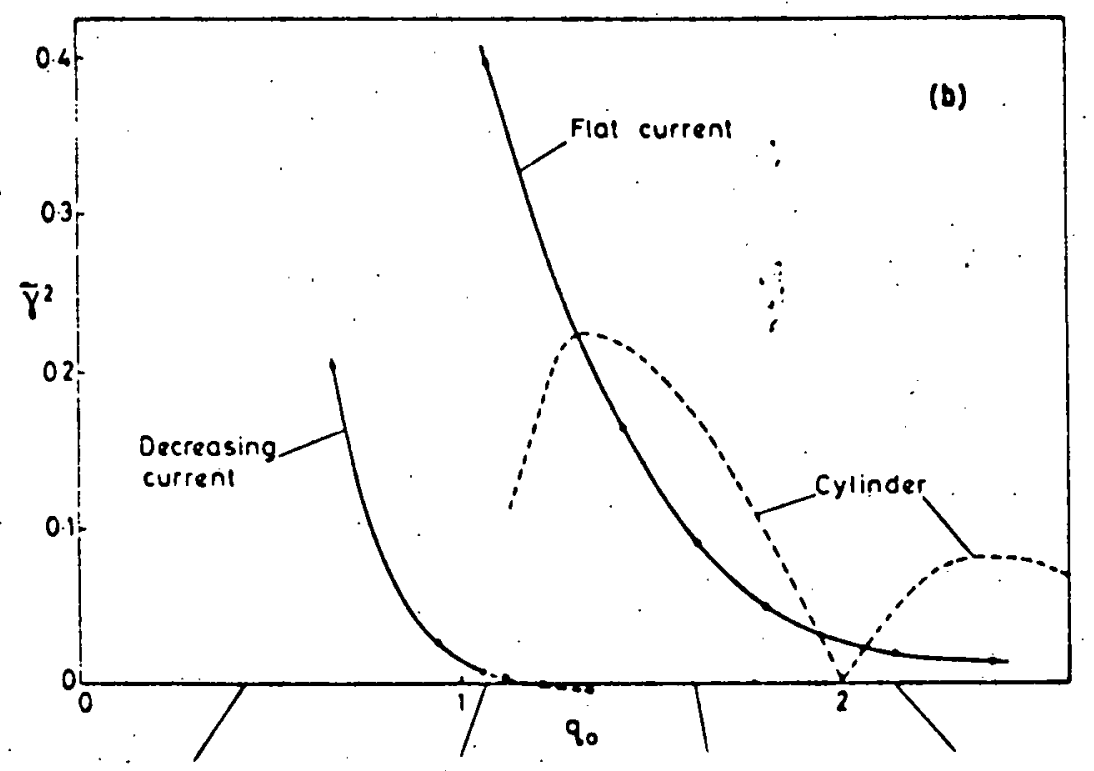

Fig. 10. Normalized growth rate for the $n=1$ external kink mode for the uniform current case and decreasing current case. 


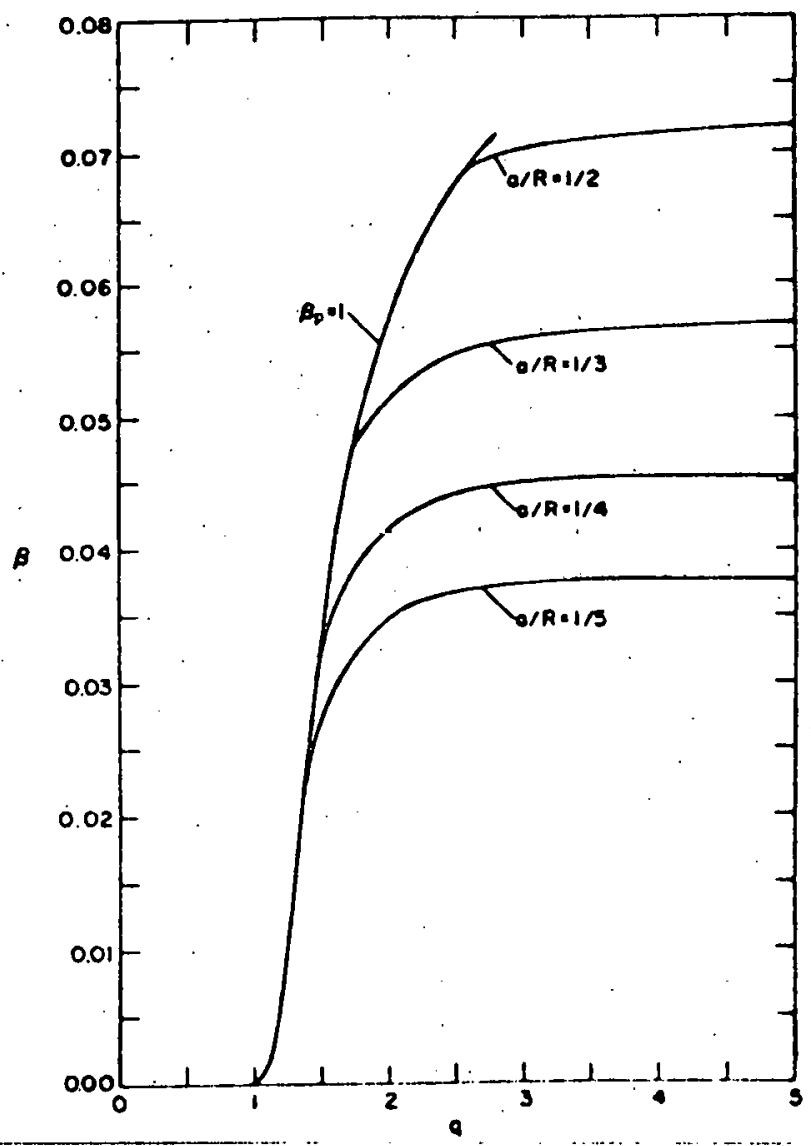

Fig. 11. Critical $\beta$ vs $q$ for the circle at various aspect ratio (Ref. 16).

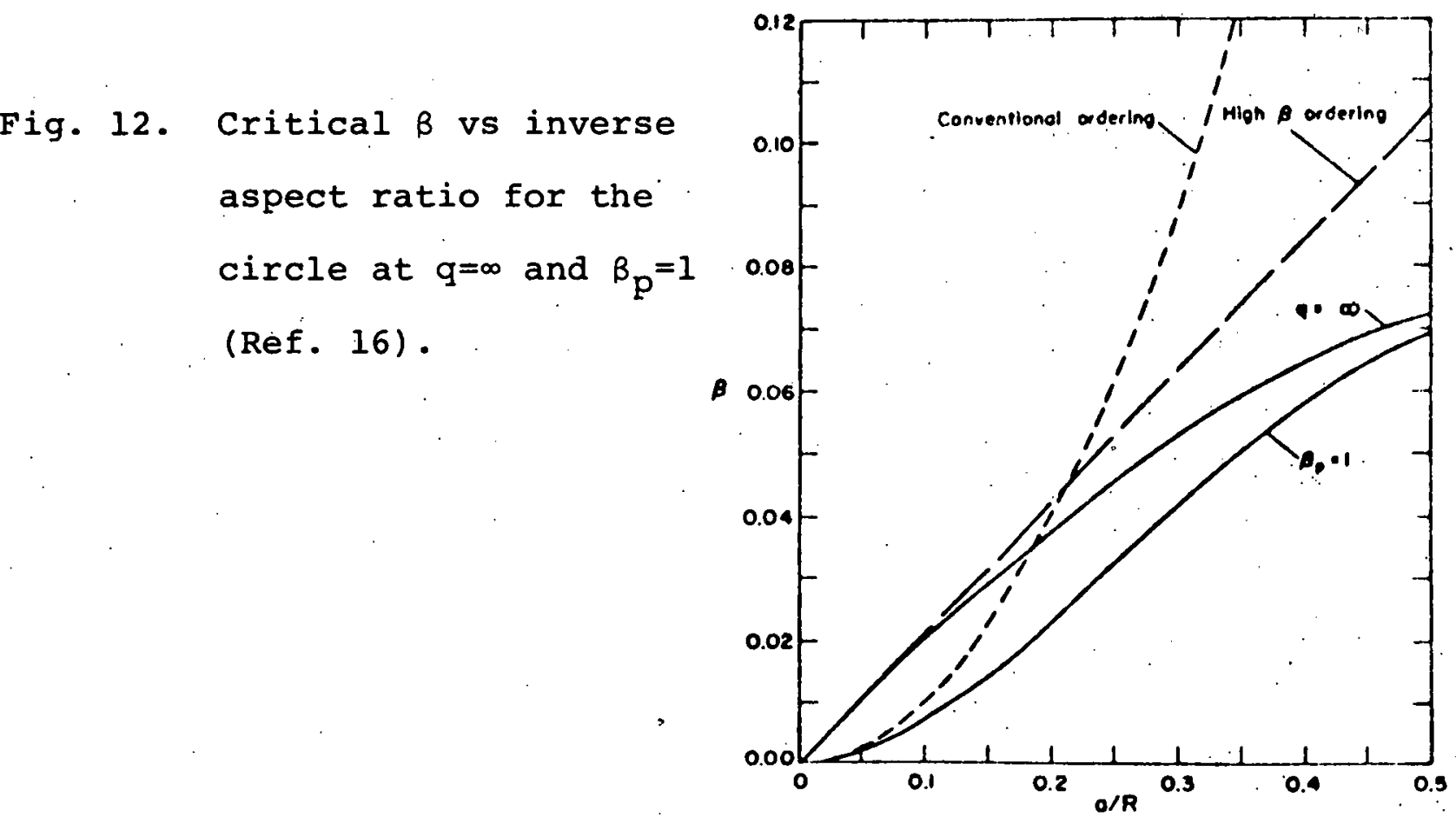




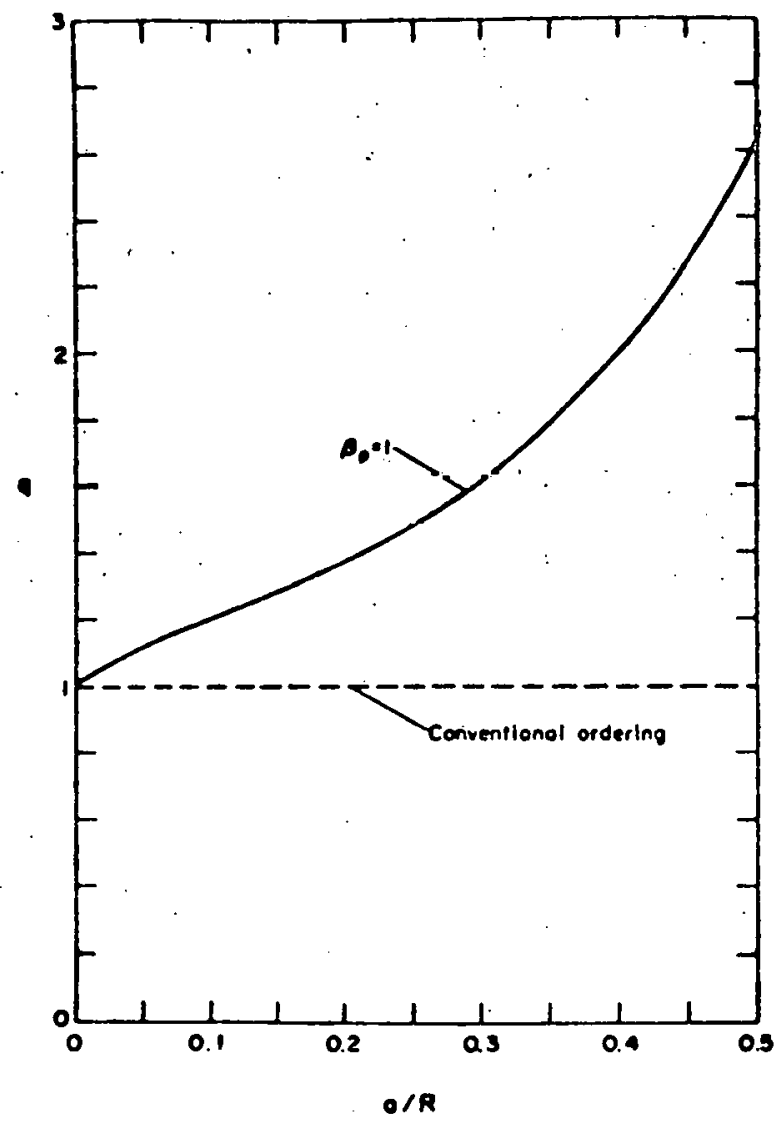

Fig. 13. Critical $q$ vs inverse aspect ratio for the circle at $\beta_{\mathrm{p}}=1$ (Ref.16).

Fig. 14. Harmonic content for the $n=1$ marginal mode (Ref. 15).

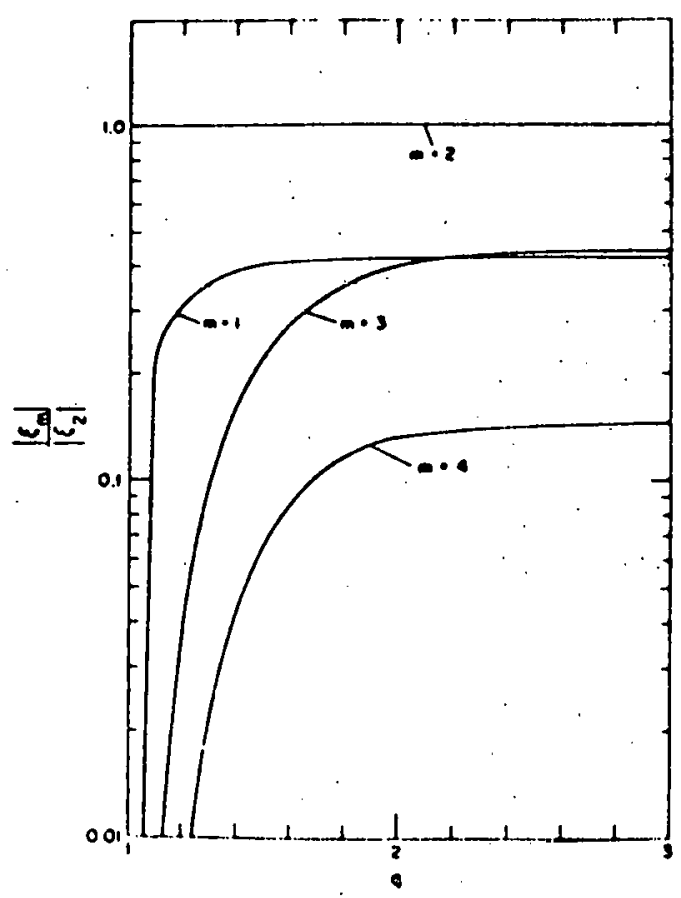




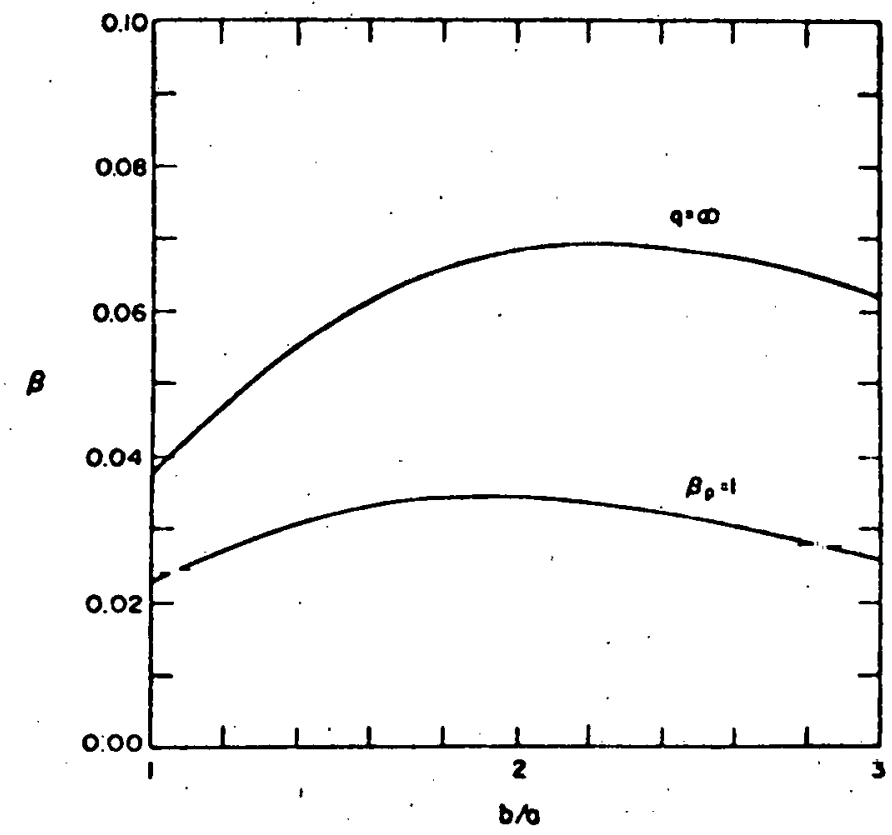

Fig. 15. Critical $\beta$ vs elongation for the ellipse at $q=\infty^{\circ}$ : and $\beta_{\mathrm{p}}=1$ for $\mathrm{R} / \mathrm{a}=5$ (Ref. 16).

Fig. 16. Optimized critical $\beta$ $\nabla s$ inverse aspect ratio for the ellipse at $q=\infty$ and $B_{p}=1$ (Ref. 16).

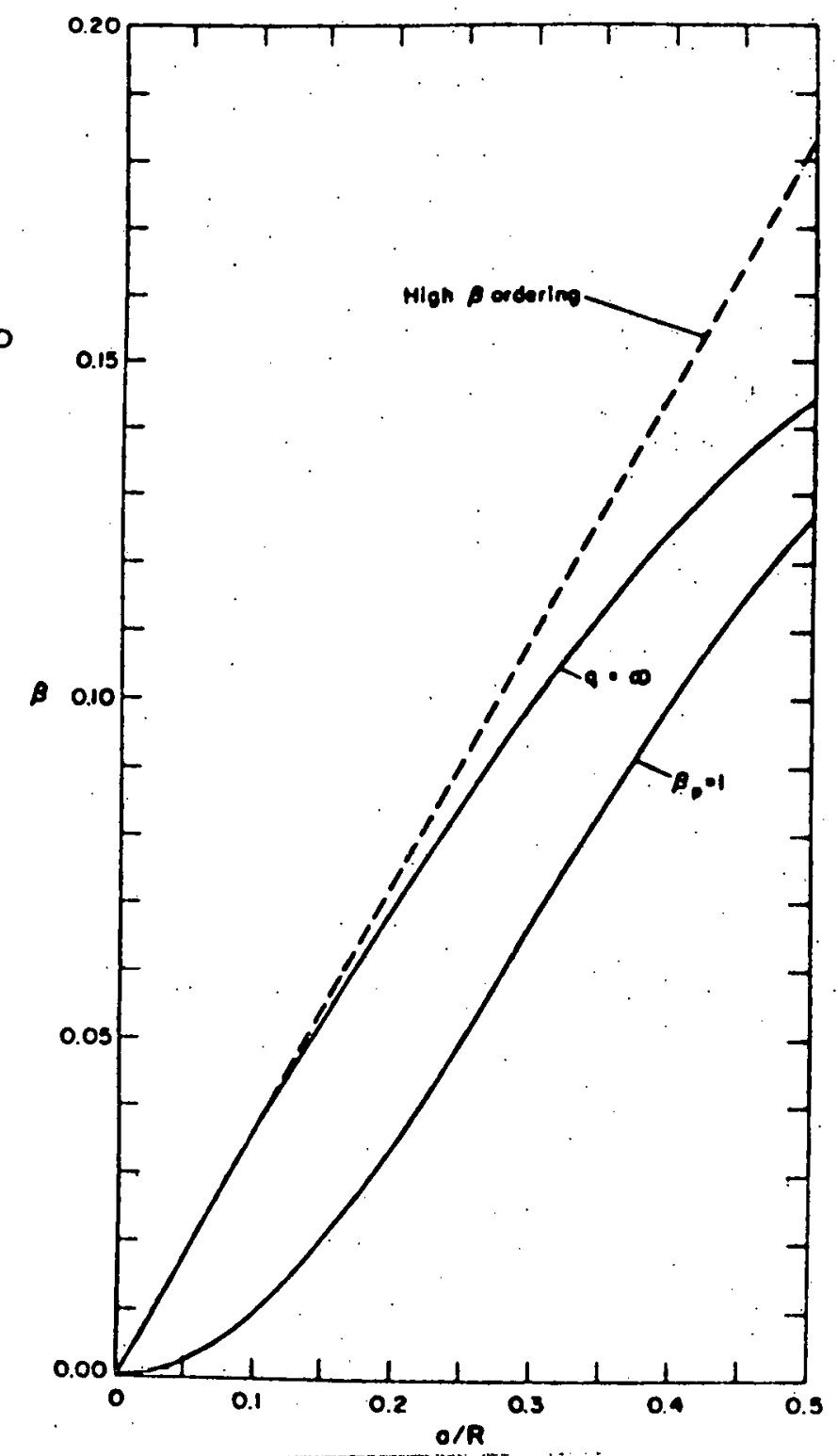




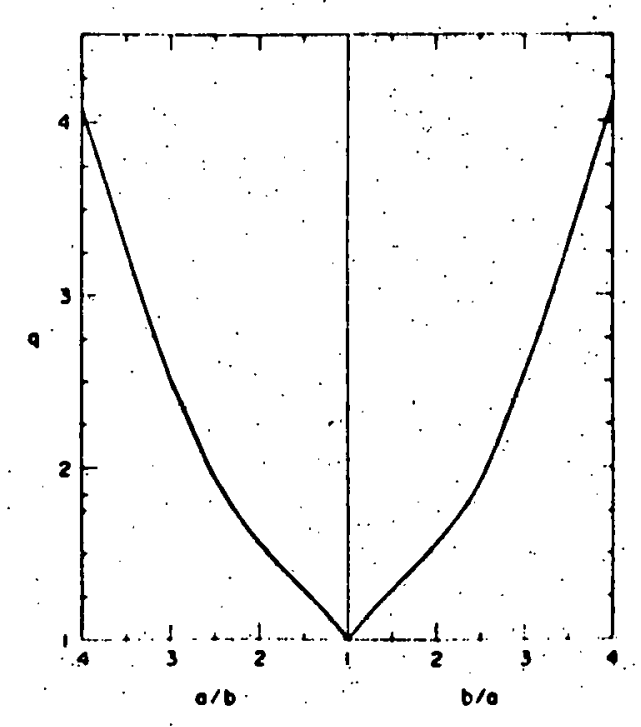

Fig. 17. Critical q vs ellipticity. for $\beta / \varepsilon \rightarrow 0 \quad(\operatorname{Ref} .17)$.

Fig. 18. Harmonic content of the marginal mode vs ellipticity.

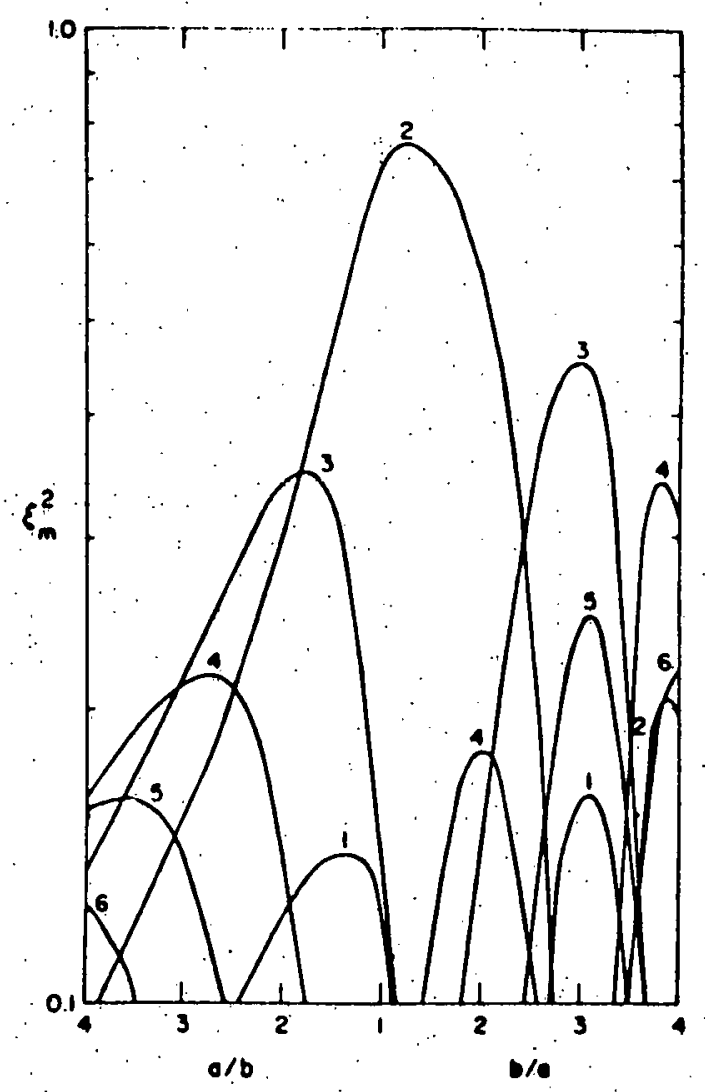




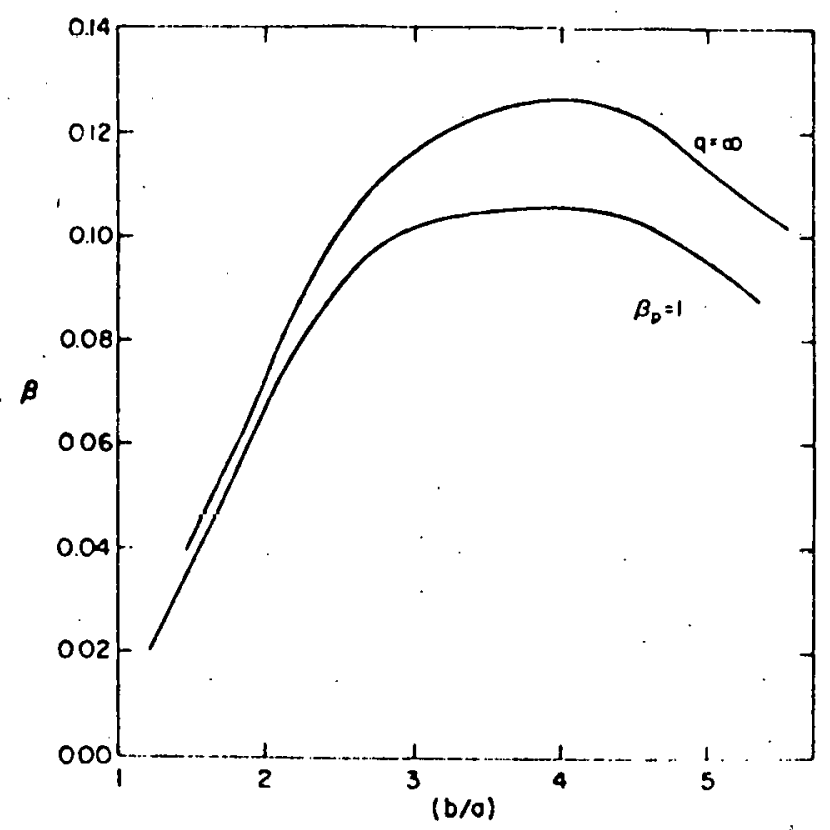

Fig.19. Critical $\beta$ vs elongation for the doublet at $q=\infty$ and $\beta_{p}=1$ for $R / a=2.44$ (Ref. 16 ).

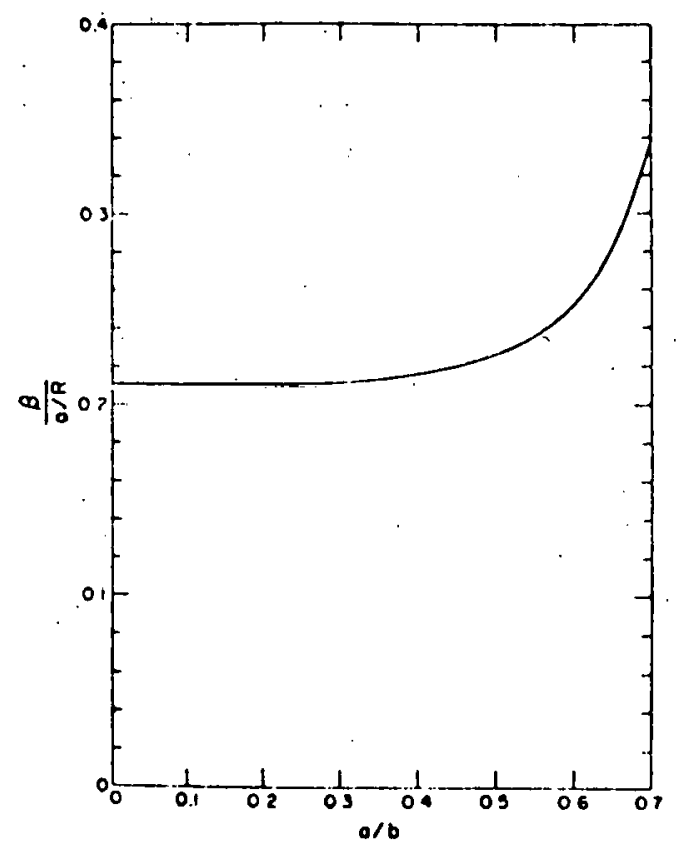

Fig. 20. Critical $\beta / \varepsilon$ vs plasmawall ratio for $n=1$ and $q=\infty \quad(\operatorname{Ref} .15)$.

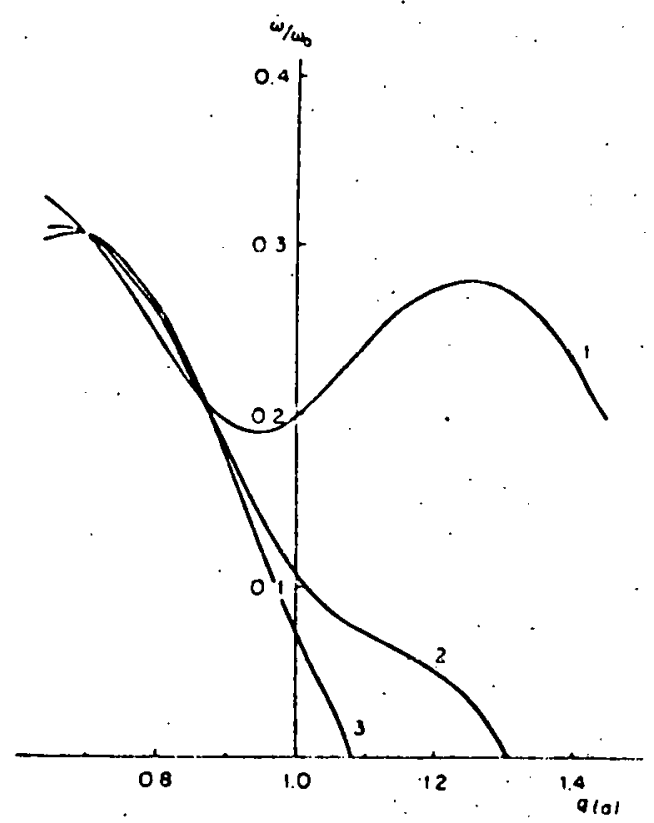

Fig. 21. Normalized growth rate of $n=1$ kink mode for plasma-wall ratio 0.625 and $R / a=10:$ 1) $\beta_{p}=3,2$ ) $\left.\beta_{p}=2,3\right) \beta_{p}=1.5(\operatorname{Ref} .20)$. 


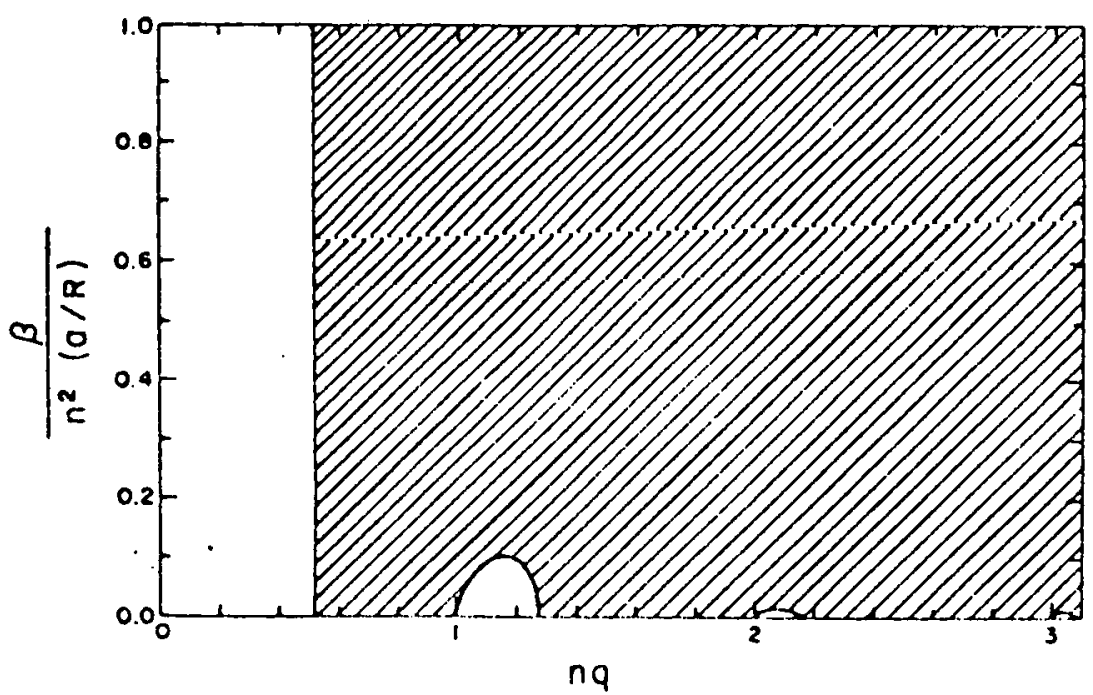

Fig.22. Critical $\beta$ for $c / a=1.4$. The shaded region is unstable (Ref. 21).

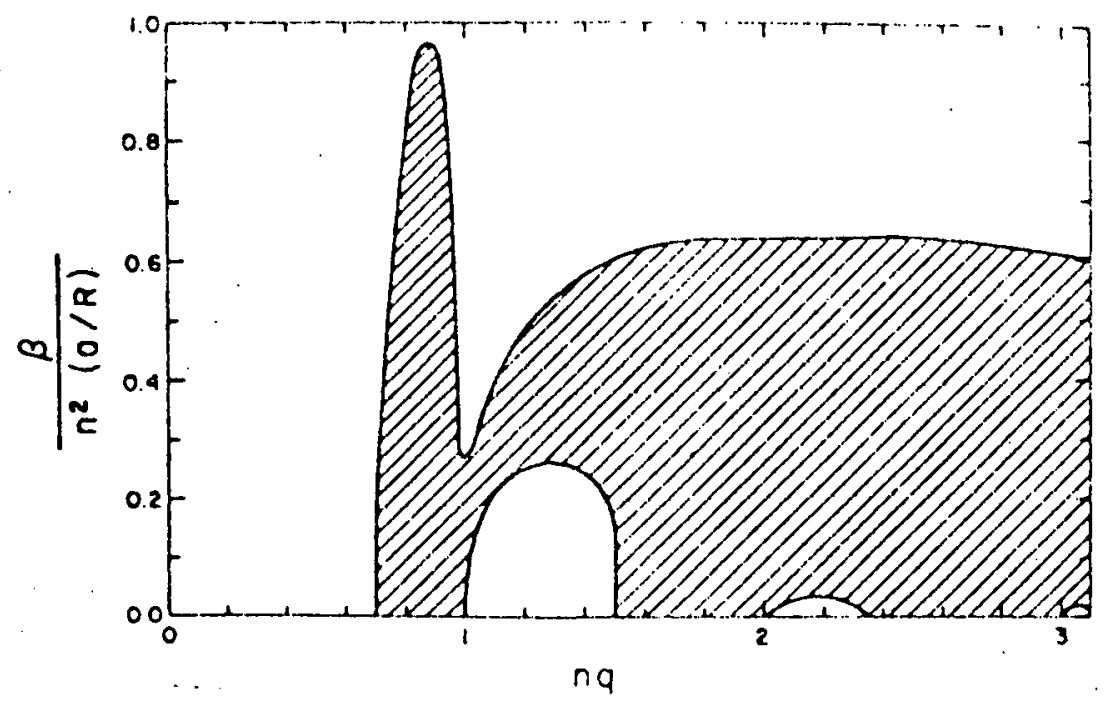

Fig. 23. Critical $\beta$ for $c / a=1.2$. The shaded region is unstable (Ref. 21). 


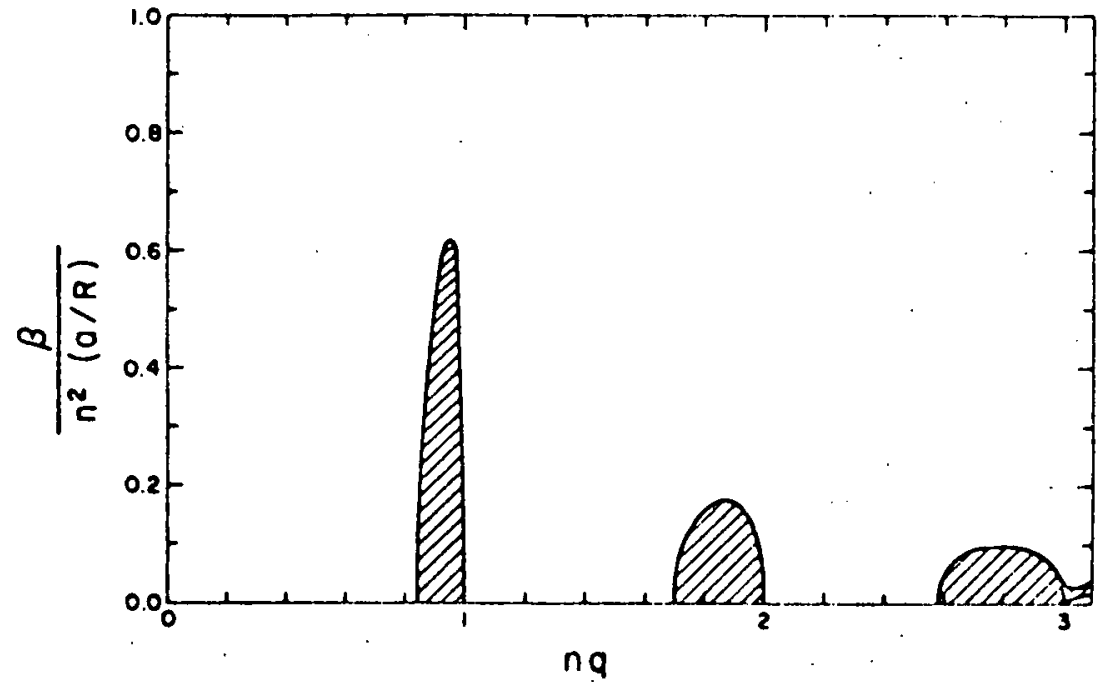

Fig. 24. Critical $\beta$ for $c / a=1.1$ The shaded region is unstable (Ref. 21). 

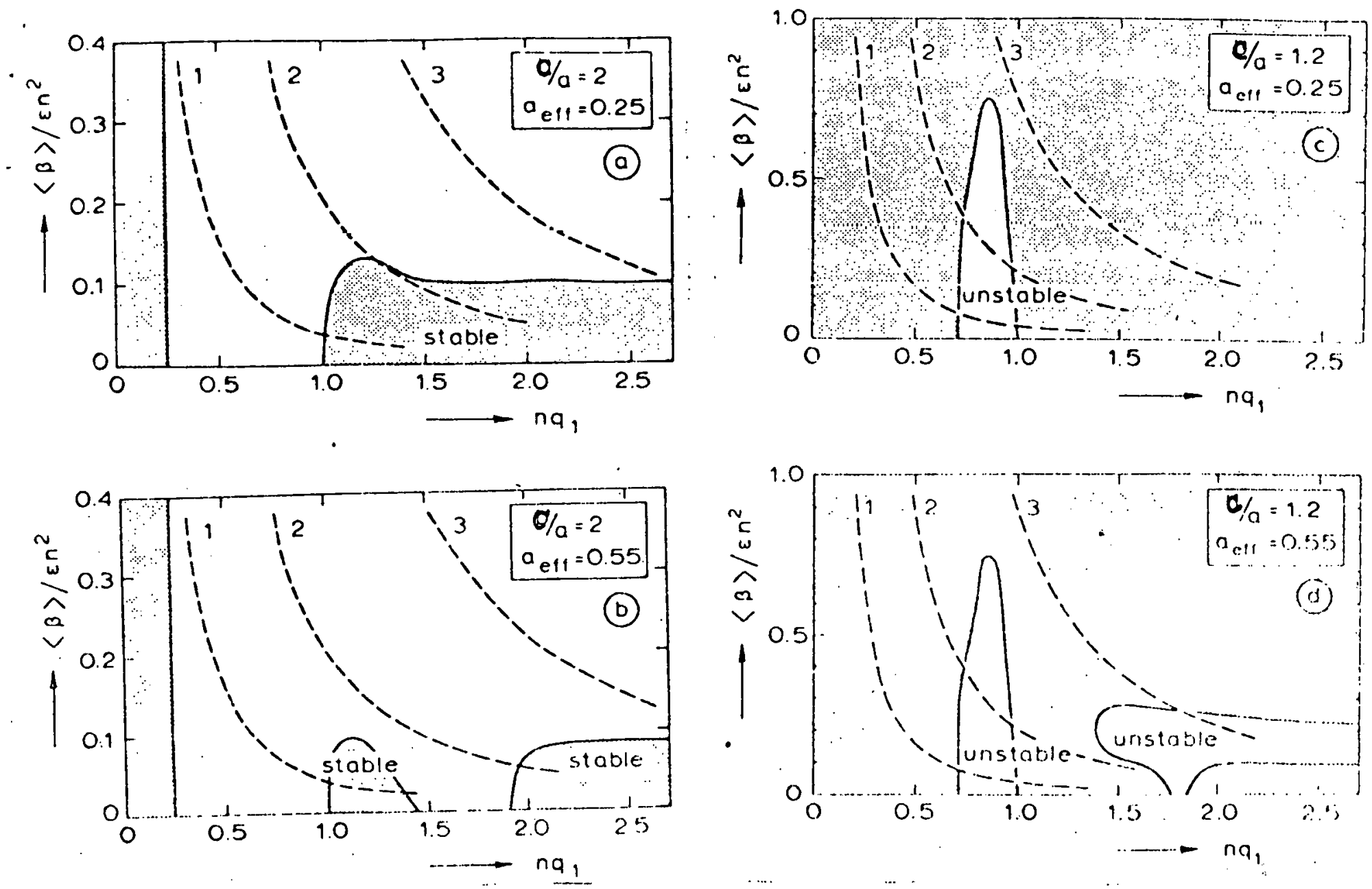

Fig.25. Stability diagrams. The dotted curves 1, 2 and 3 represent families of equilibria with fixed values of $\delta$ and $\mathrm{q}_{1} / \mathrm{q}_{0}$

(a) 1. $\delta=0.02, q_{1} / q_{0}=14.8$

2. $\delta=0.1, \mathrm{q}_{\mathrm{i}} / \mathrm{q}_{0}=15.2$

3. $\delta=0.25, q_{1} / q_{0}=18.2$

(b) $1 . \quad \delta=0.02, q_{i} / q_{0}=2.3$

2. $\delta=0.1, q_{1} / q_{0}=2.4$

3. $\delta=0.25, \mathrm{q}_{\mathrm{i}} / \mathrm{q}_{0}=3.1$,

(c) as in (a)

(d) as in (b) (Ref. 22). 


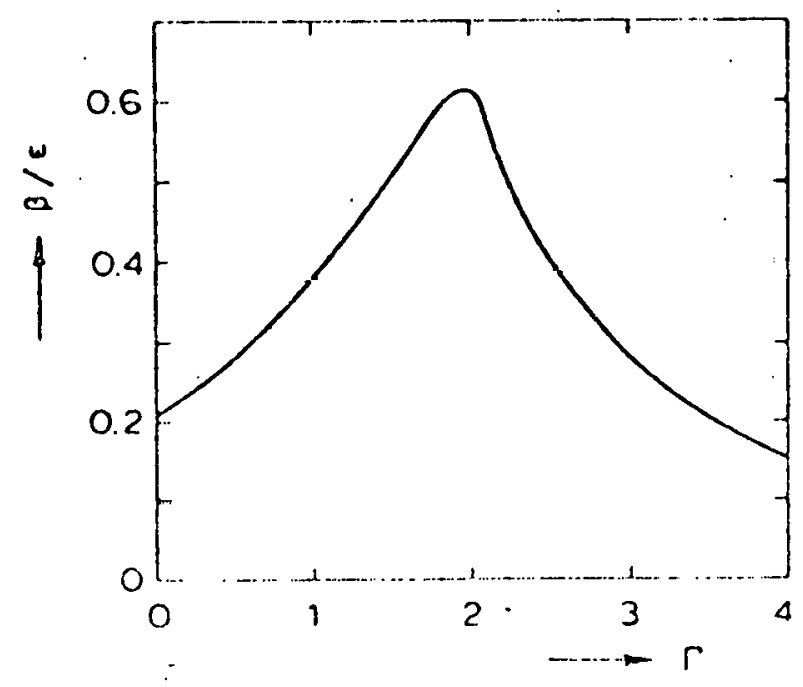

Fig.26. Optimum $\beta / \varepsilon$ vs $\Gamma$ (Ref. 26).

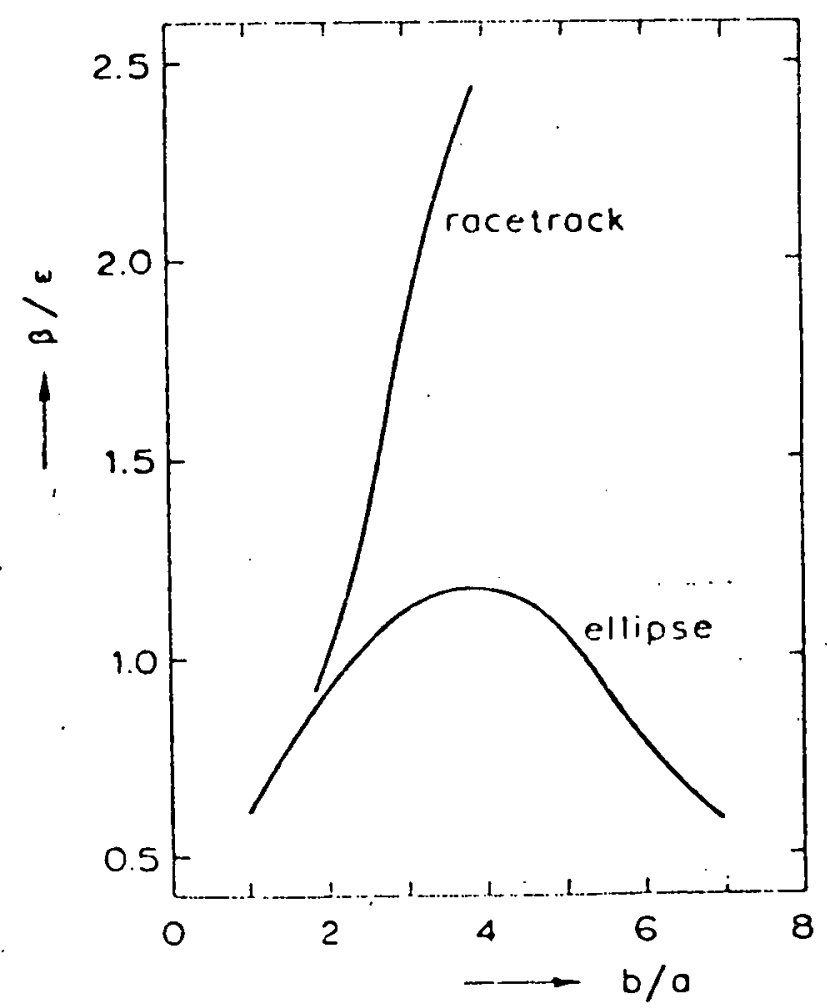

Fig.27. Maximum $\beta / \varepsilon$ vs elongation b/a (Ref. 26). 


\section{MHD Stability of Internal Kink Modes}

First we consider an analysis in a cylindrical approximation. Within a cylindrical analysis and for monotonically decreasing current profiles, kink instabilities separate into two classes: ${ }^{1)}$ 'external' kink modes (which are treated in §2) which have singular surfaces (where $\vec{k} \cdot \vec{B}=m B_{\theta}+k r B_{z}=0$ ) falling into the vacuum region outside the plasma; and internal kink modes (mainly $m=1$ ) which have their singular surfaces falling into the perfectly conducting region. The internal $m=1$ kink mode occurs whenever the safety factor $q(x)$ falls below unity (for monotonic current profiles, on the axis $r=0$ ). The energy reservoir and growth rate for this mode are smaller by $\mathrm{B}_{\theta}^{2} / \mathrm{B}_{\mathrm{Z}}^{2} \sim \varepsilon^{2}$ compared with those for the external kink modes. Here we consider ${ }^{1}$ ) a perfectly conducting plasma column. of pressure $\mathrm{p}(\mathrm{x})$ in an axial magnetic field $\mathrm{B}_{\mathbf{z}}(r)$ and azimuthal magnetic field $B_{\theta}(r)$ and with a fixed conducting wall at $r=b$.

The potential energy for perturbations with radial displacement $\xi(r) \cos (m \theta+k z)$ is

$$
W=\frac{1}{8} \int_{0}^{b}\left[f\left(\frac{d \xi}{d r}\right)^{2}+g \xi^{2}\right] d r
$$

where

$$
\begin{gathered}
f=\frac{r^{3}(\vec{k} \cdot \vec{B})^{2}}{m^{2}+k^{2} r^{2}}, \\
g=\frac{\left(m^{2}-1\right)^{r}(\vec{k} \cdot \vec{B})^{2}}{m^{2}+k^{2} r^{2}}+\frac{k^{2} r^{2}}{m^{2}+k^{2} r^{2}}\left(8 \pi p^{\prime}+r(\vec{k} \cdot \vec{B})^{2}+\frac{2}{r} \frac{k^{2} r^{2} B z^{2}-m^{2} B_{\theta}^{2}}{m^{2}+k^{2} r^{2}}\right) .
\end{gathered}
$$


The limit $k_{z} r<1$ is considered. For the special case of $m=1$, the first term in eq. (3) vanishes and $g$ is reduced to terms of order $k^{2} r^{2} v \varepsilon^{2}$. To order $k^{2} r^{2}, w$ is minimized by the singuiàr function

$$
\xi(r)= \begin{cases}\xi_{a}=\text { const } & r<S \\ 0 & x>S\end{cases}
$$

where $S$ is the singular surface. The minimum is

$$
W_{\min }=\frac{1}{8} \xi_{a}^{2} \int_{0}^{s} g_{1} d r
$$

where $g_{1}$ is the value given by eq. (3) for $m=1$ and

$$
g_{1}=k^{2} r\left(8 \pi r p^{1}+3 k^{2} r^{2} B_{z}^{2}+2 k r B_{z} B_{\theta}-B_{\theta}^{2}\right)
$$

correct to order $\mathrm{k}^{2} \mathrm{r}^{2}$.

For monotonically decreasing profiles of pressure $p(r)$ and current $j_{z}(r)$, we have $g_{1}<0$ for $0<r<s$; hence, $w_{\min }<0$ and instability occurs. The above analysis shows that the stability criterion for the internal $\mathrm{m}=1 \mathrm{kink}$ mode is $q(0)>1$.

When $q(0)<1$, other modes with $(m, n)$ and $q(0)<m / n<1$ are possible. For $m, n>>1$, these modes become localized near the singular surface $q=m / n$ and are flute-like. ${ }^{3}$ ) Suydam criterion applies for these modes. In toroidal geometry with a nearly circular cross-section, the stability criterion for the flute modes is also $q(0)>1,2$ ) which is the 
same; as for the internal $m=1$ kink mode in cylindrical geometry. Therefore there is a strong relation between the internal $\mathrm{m}=1$ kink mode and the flute (or interchange) modes.

Another interesting feature is found in an elliptic cylindrical tokamak. For a circular cylinder the destabilizing forces for the internal $\mathrm{m}=\mathrm{l} \mathrm{kink}$ mode are not those which destabilize the external kink modes in the long-wavelength limit $\left(k_{1}<<1\right)$. If one retains only these effects, the internal $m=1$ kink mode is marginally stable in a circular cylindrical plasma (see eqs. (5) and (6), $g_{1} \rightarrow 0$ as $\mathrm{k}^{2} \mathrm{r}^{2} \rightarrow 0$ ). The internal kink mode becomes unstable only if one includes smaller destabilizing terms which are related in the pressure gradient, the magnetic curvature and finite axial wavelength. It remains to be seen what happens to the internal $m=1$ mode within the external kink modes ordering when the plasma cross-section is no longer circular. Laval ${ }^{4}$ ) showed that the internal $\mathrm{m}=1 \mathrm{kink}$ mode is no longer marginally stable at the lowest order of the long-wavelength expansion, so that the smaller destabilizing terms are no longer important. For a weakly elliptical crosssection cylindrical plasma, Laval4) found that the internal $m=1$ kink mode becomes unstable as soon as $q(0)<1$ on the magnetic axis. (In contrast to circular cylinder, one needs not consider higher order terms beyond $\mathrm{k}^{2} \mathrm{r}^{2}$.) Edery et al ${ }^{5}$ ) extended the analyses, and found that elliptical cylinders, which are small distortions of a circular cylindrical plasma, are destabilizing for the internal $m=1$ kink mode; however, 
triangular and square distortions are stabilizing for the internal $\mathrm{m}=1 \mathrm{kink}$ mode.

Here it should be noted that certain non-circular crosssections can improve the stability against localized flute mores. Those results demonstrate that the Mercier stability criterion ${ }^{6}$ ) for flute modes is not sufficient to prevent the internal m=l kink modes. It is not clear whether the Mercier stability criterion is valid for low $m(m=2,3,4)$ internal kink modes or not, since it is derived on the assumption that $m \gg>1, n>>1$ but $m / n$ is finite.

Bussac et al. ${ }^{7}$ ' gave the stability criterion of the internal $\mathrm{m}=1 \mathrm{kink}$ mode in the toroidal geometry for plasmas with circular cross-section. Contrary to the above results in cylindrical geometry, the internal $m=1$ kink mode can be stable if the pressure gradient is sufficiently low. This result is of interest to us in the stability of the internal $m=1$ kink mode in real tokamak plasmas.

Numerical computations have demonstrated significant effects due to the toroidal geometry on the stability of the internal $\mathrm{m}=1 \mathrm{kink}$ mode. Sykes and Wesson ${ }^{8}$ ) showed that instability has been removed for $q_{0}>0.55$ for $m=1, n=2$ and $q_{0}>0.73$ for $m=2$ and $n=2$ and the fundamental toroidal mode, $\mathrm{n}=1$, is stable $(\mathrm{a} / \mathrm{R}=1 / 3)$. Schneider and Bateman $\left.{ }^{9}\right)$ also showed that for a square cross-section toroidal plasms, the instability with $m=1$ and $n=1$ could not be found $(a / R=1 / 2)$. Recently Berger et al. ${ }^{10}$ ) investigated internal kink modes 
in the toroidal geometry by use of the equilibrium configuration given by eq. (15) in \$2. For the circular cross-section tokamak, the growth rates of the most unstable modes are shown in Fig. $1-3$ as functions of $q(0)$ for $n=2,3$ and 4 . For $n=1$ $\mathrm{n} n$ unstable modes are found. The azimuthal mode number $\mathrm{m}$ is introduced as an integer, which reduces to the usual azimuthal mode number in straight circular geometry. On the figures the range of $q(0)$, for which the singular surface $\mathrm{nq}(\psi)=\mathrm{m}$ lies inside the plasma, is shown by the segment identified by $m$. The stability limit remains below the Mercier criterion $q(0)=1$. The limit increases with $n$, and probably accumulates at $q(0)=1$ as $n \rightarrow \infty$. Figure 4-6 shows the results for an elliptical toroidal plasma. They are analogous to the circular toroidal plasma $(E=1)$. All the modes are strongly destabilized. There is now an unstable $\mathrm{n}=1$ mode. In Fig. 4-6 the presence of the peak with dominantly $m=n$ pushes the stability limit to $q(0) \approx_{1} .1$. These results show that in the toroidal geometry if $q(0)$ is lowered, it is the high $\mathrm{m}$ and high $\mathrm{n}$ fixed boundary modes which become first unstable.

Finally we should note ballooning modes which may provide the stringent limitation on the maximum $\beta$ value of high $\beta$ tokamak. When the interchange (or flute) modes (displacement vector constant along field lines) are stable, the ballooning modes may appear, especially in high $\beta$ regime. Ballooning modes are highly localized modes, nonconstant along field 
lines, especially with large displacements near the outer radius; typical displacements are shown in Fig. 7.11) Relaxing the constraint on the displacement vector allows the unfavorable curvature to drive thio mode unstable. The ballooning modes provide a mechanism for moving plasma from the center to the outer surface and are not completely stabilized by the conducting wall. ${ }^{13)}$ When the external kink modes and internal kink modes are stabilized, the maximum $\beta$ value may be governed by the ballooning modes. More analytical and numerical studies are required for various tokamak configurations to know the relation between the flute-like modes and the internal low $\mathrm{m}$ kink modes and which mode is most important in real tokamak experiments. 


\section{References of $\$ 3$}

1. V.D. Shafranov: Sov. Phys-Technical Physics 15, 175 (1970).

2. V.D. Shafranov and E.I. Yurchenko: Soviet Phys-JETP 26, 682 (1968).

3. B.R. Suydham: Proc. U.N. Int. Conf. Peaceful Uses At. En., Geneva 1958 Vol. 31 pp. 157-159 Columbia U. Press 1959.

4. G. Laval: Phys. Rev. Lett. 34, 1316 (1975).

5. D. Edery, G. Saverl, R. Pellat and J.L. Soul: Phys. Fluids 19, 260 (1976).

6. G. Mercier: Nucl. Fusion 1, 47 (1960).

7. M. Bussac et al.: Phys. Rev. Letts. 35. 1638 (1975).

8. A. Sykes and J.A. Wesson: 5th IAEA Conference on Plasma Physics and Controlled Nuclear Fusion Research (Tokyo, 1974), $\mathrm{CN}-33 / \mathrm{Al} 2-3$.

9. W. Schneider and G. Bateman: 5th IAEA Conference on Plasma Physics and Controlled Nuclear Fusion Research (Tokyo, 1974 ), $\mathrm{CN}-33 / \mathrm{Al} 2-2$.

10. D. Berger, L.C. Bernard, R. Gruber and F. Troyan: 6th IAEA Conference on Plasma Physics and Controlled Nuclear Fusion Research (Berchtesgaden), (1976).

11. A.M.M. Todd et al: MATT - (Princeton University) 1977. q 


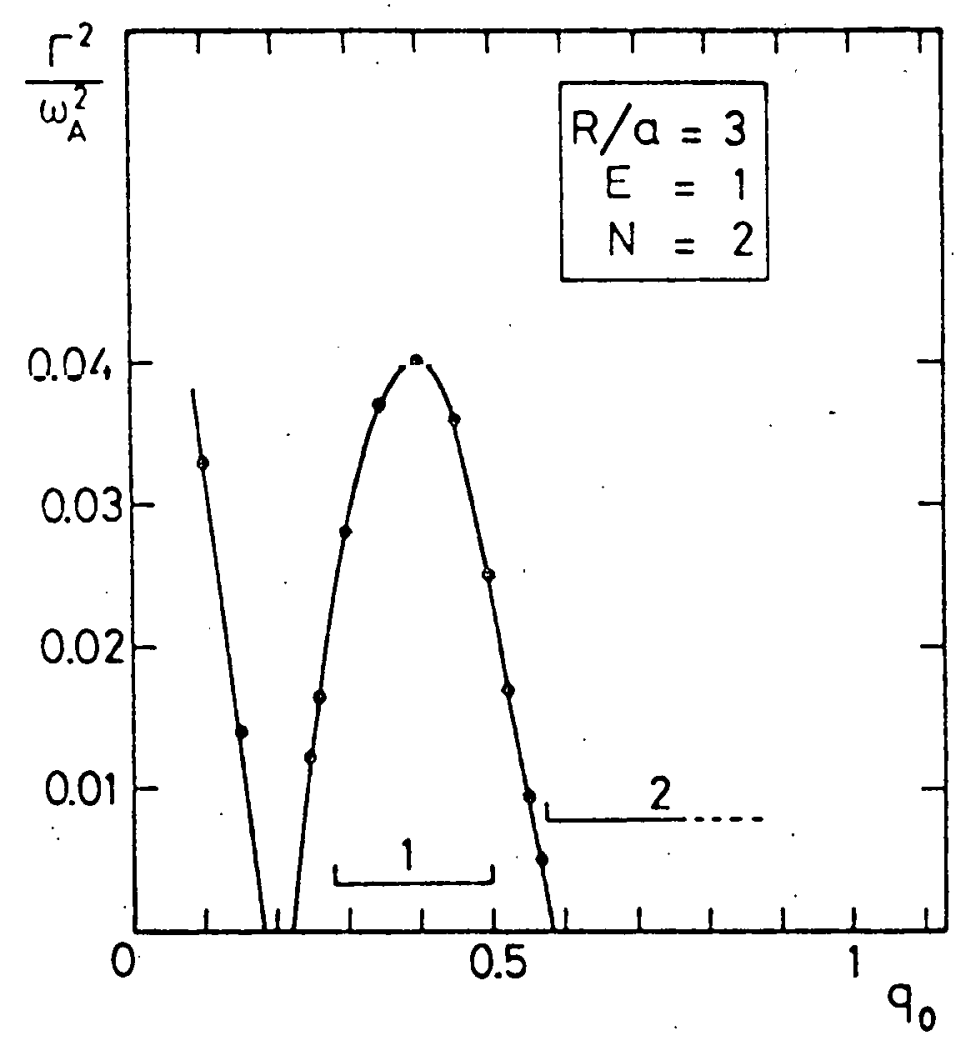

Fig. 1

The normalized growth rate of the most unstable modes versus the safety factor on the magnetic axis $q_{0}$. Fixed boundary case. $E=1$ and $n=2 \quad(\operatorname{Ref} .7)$.

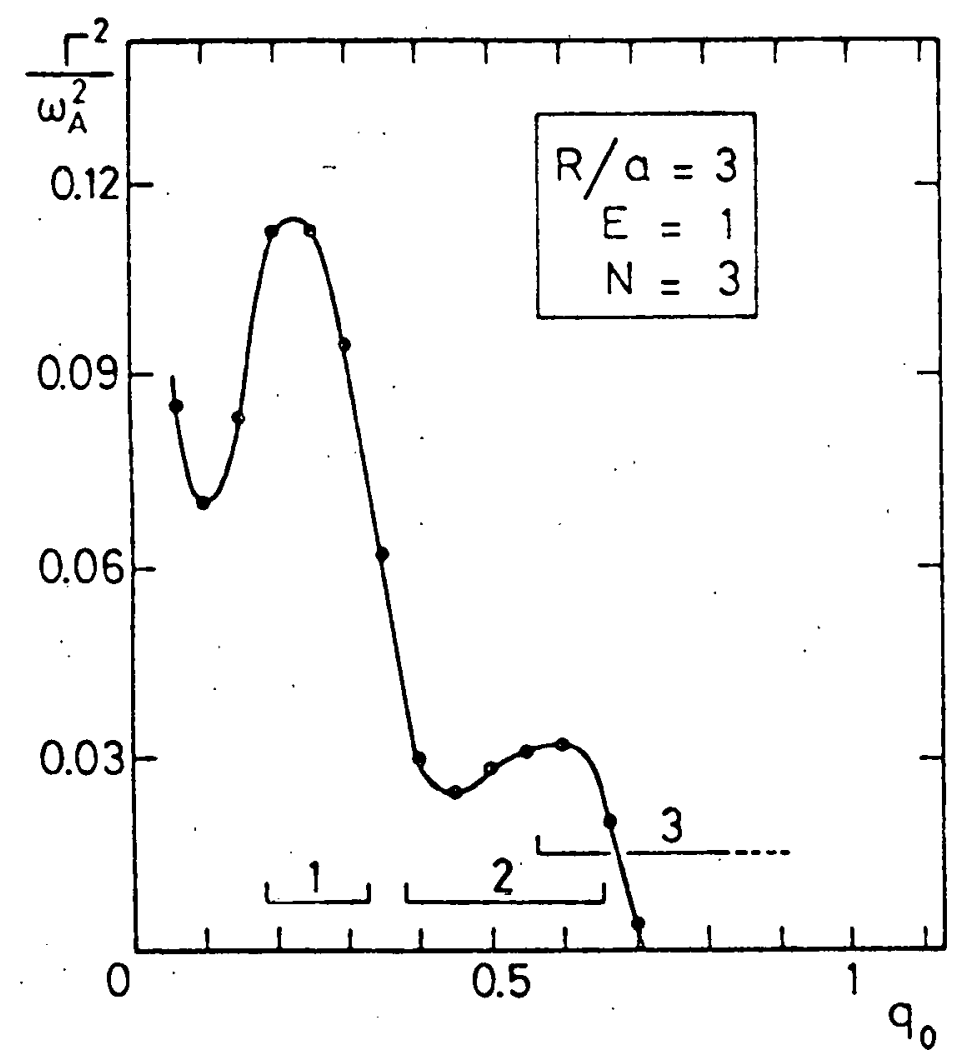

Fig. 2

$E=1, n=3$ (Ref. 7) 


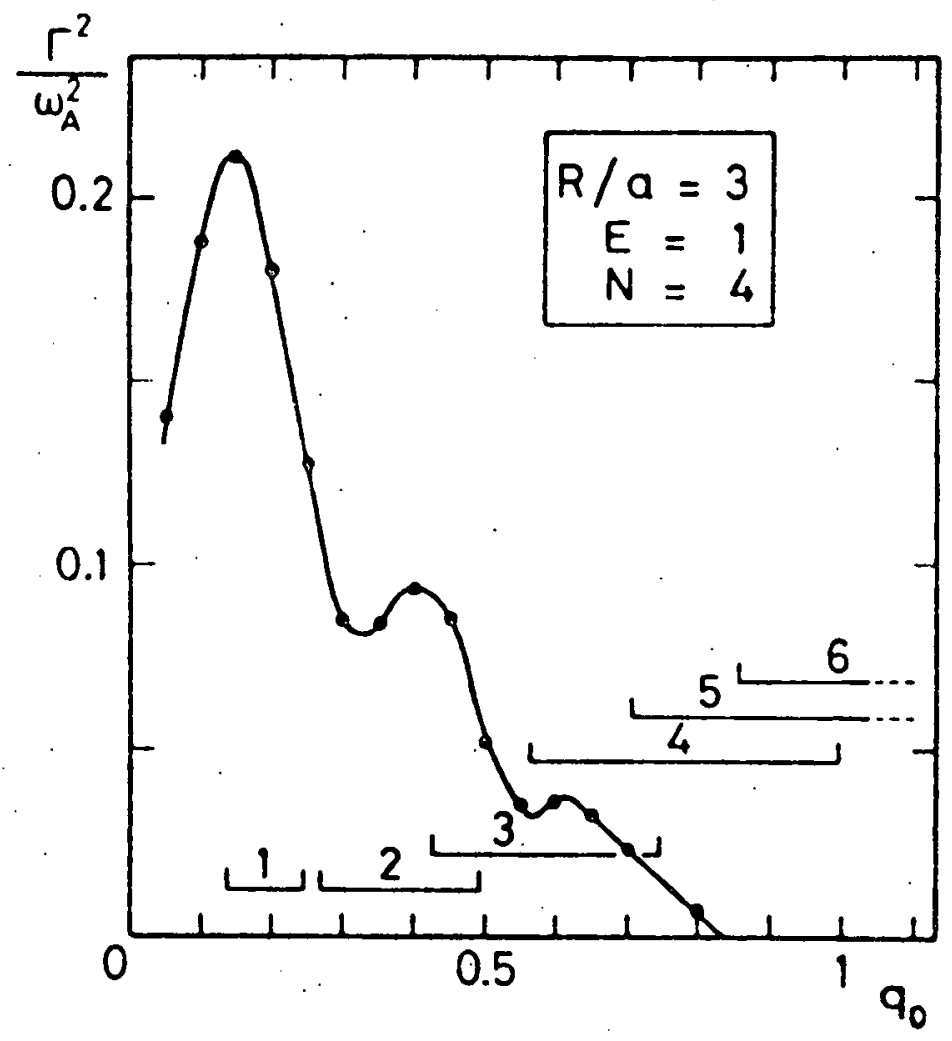

Fig. 3

$E=1, n=r \quad(\operatorname{Ref} .7)$.

Fig. 4

The normalized growth

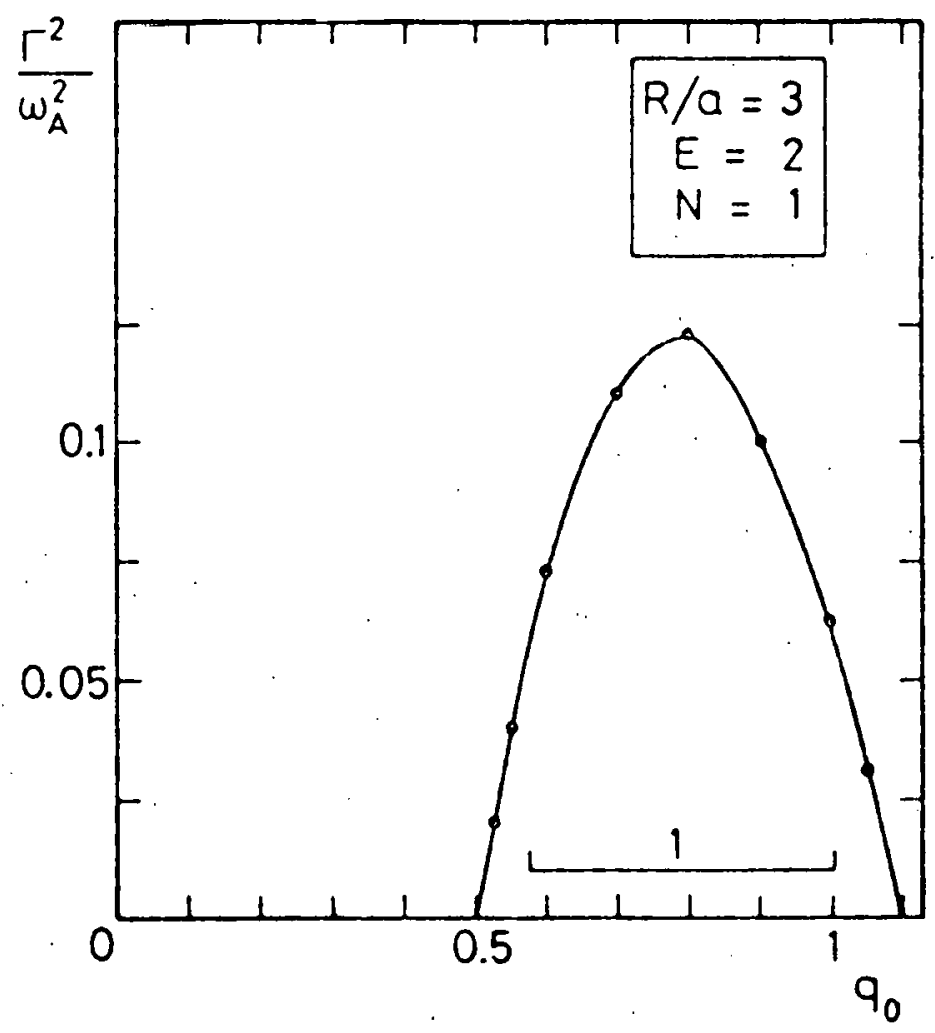

rate of the most unstable modes versus $q_{0}$. Fixed boundary case. $E=2$ and $n=1$ (Ref. 7). 


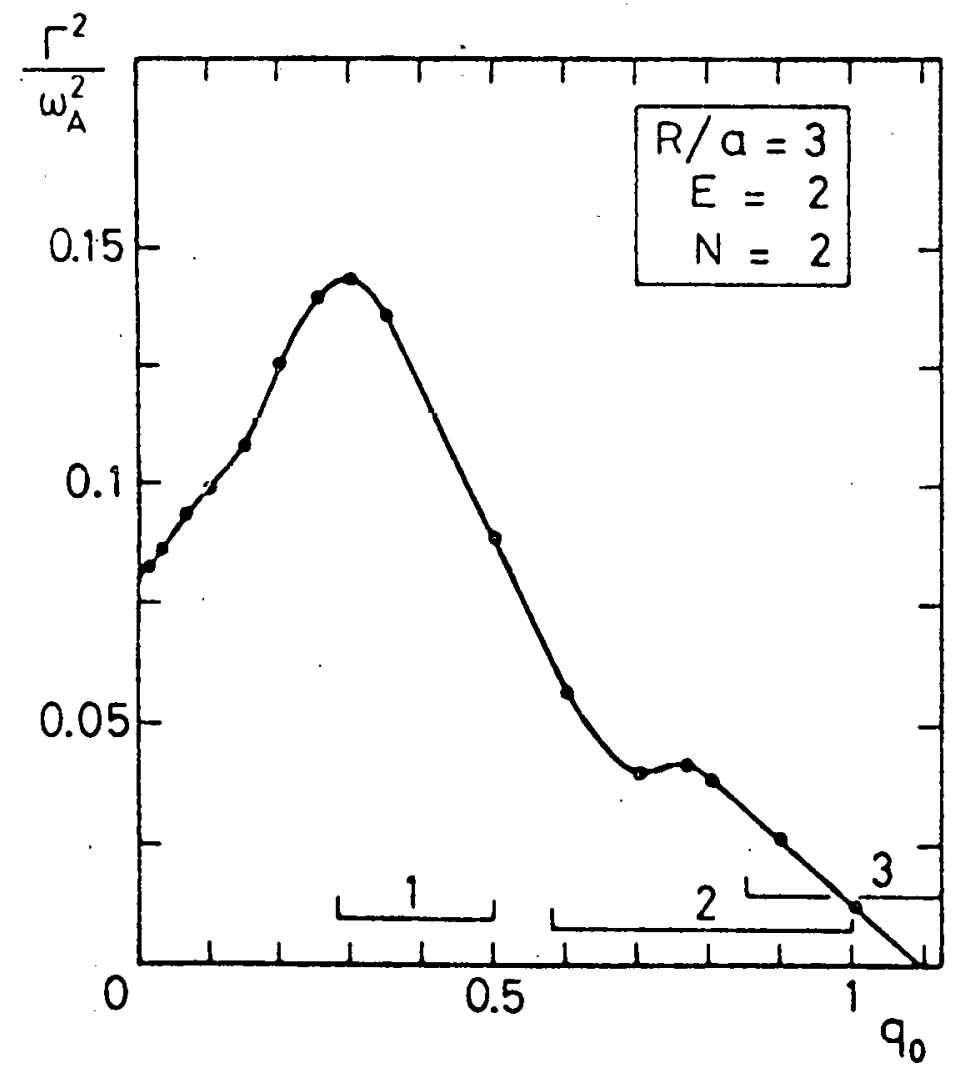

Fig. 5

$E=2, n=2 \quad(\operatorname{Ref} \cdot 7)$.

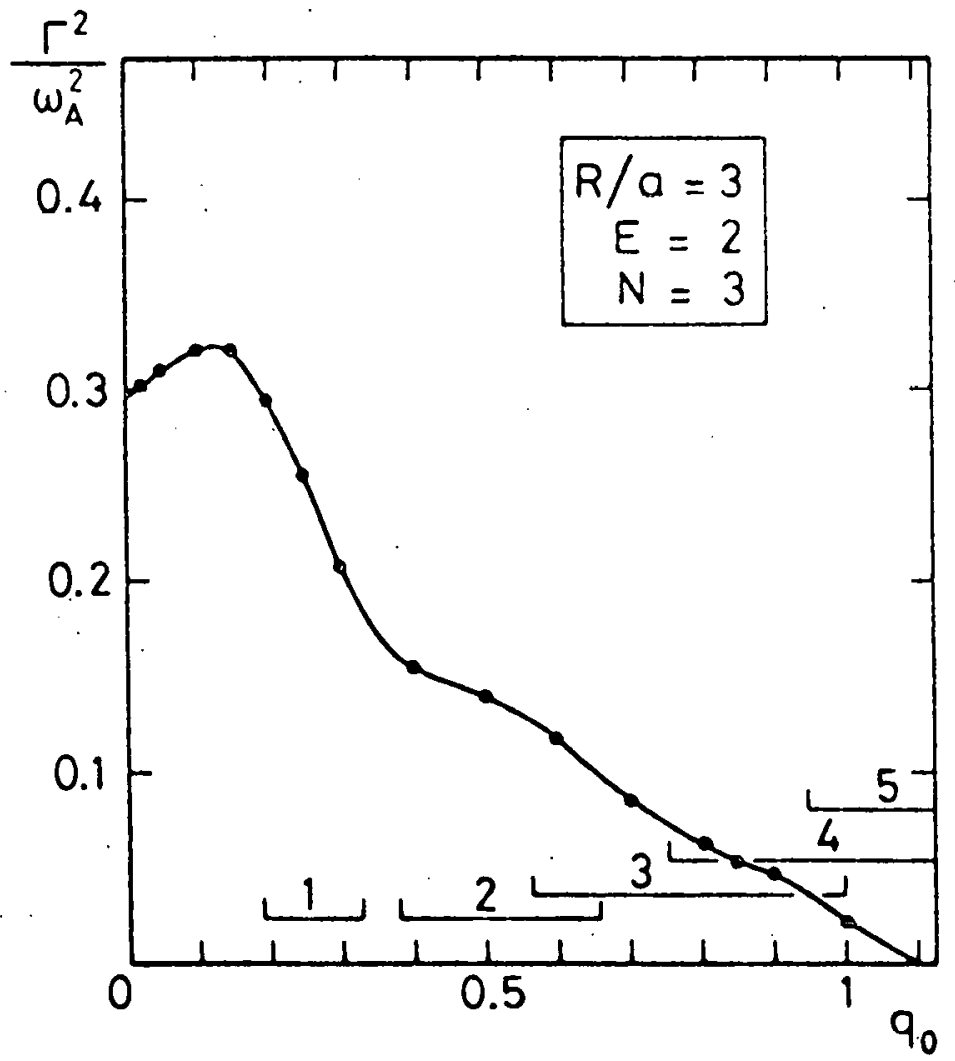

Fig. 6

$E=2, n=3(\operatorname{Ref} .7)$. 
Fig. 7. Displacement vector for the fixed boundary $n=3$ mode at $\beta^{*}=3.0 \%$, where $\beta^{*}$ denotes the fusion power averaged pressure ${ }^{11}$ )

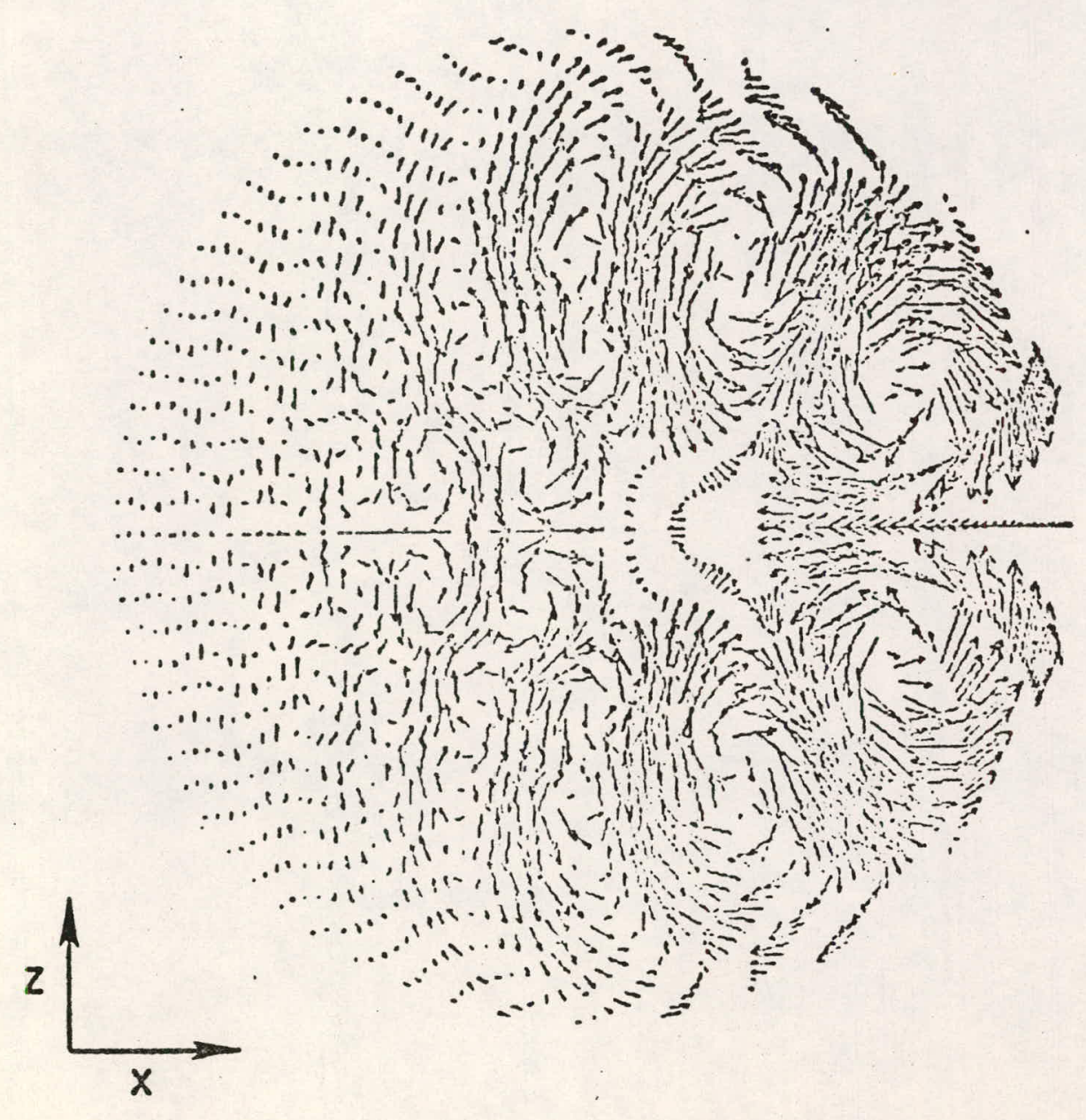


Acknowledgements

'l'he author gratefully acknowledges Professor Chu for valuable discussions and Dr. Lui and Dr. Johnston for encouragement. 